\title{
SQUARE-INTEGRABLE FACTOR REPRESENTATIONS OF LOCALLY COMPACT GROUPS
}

\author{
BY \\ JONATHAN ROSENBERG $\left({ }^{1}\right)$
}

\begin{abstract}
ABSTRACr. The well-known theory of square-integrable representations is generalized to the case of primary representations (not necessarily type I) quasi-contained in either the regular representation or the representation induced from a character of the center of a (not necessarily unimodular) locally compact group, and relations with the topology of the primitive ideal space of the group $C^{*}$-algebra are obtained. The cases of discrete and almost connected groups are examined in more detail, and it is shown that for such groups, square-integrable factor representations must be traceable. For connected Lie groups, these representations can (in principle) be determined up to quasi-equivalence using a complicated construction of $L$. Pukanszky-for type I simply connected solvable Lie groups, the characterization reduces to that conjectured by C. C. Moore and J. Wolf. In the case of unimodular exponential groups, essentially everything is as in the nilpotent case (including a result on multiplicities in the decomposition of $L^{2}(G / \Gamma), \Gamma$ a discrete uniform subgroup of $\left.G\right)$. Finally, it is shown that the same criterion as for type I solvable Lie groups characterizes the squareintegrable representations of certain solvable $\mathfrak{b}$-adic groups studied by $\mathbf{R}$. Howe.
\end{abstract}

1. Introduction. The theory of square-integrable irreducible representations of unimodular groups has been understood for some time, and is easily accessible in [8, \$14] and [36]. Modifications have been introduced to take into account groups with noncompact center (e.g., [24]) and nonunimodular groups (most notably [10]; cf. also [25], [26]), but until recently, we were unaware of any treatment of non-type I square-integrable representations aside from some remarks on cyclic representations in [36], [25], and [26]. A few months ago, the author received a copy of [7], which, in Chapter III, does treat a much more general situation, but here the emphasis is on von Neumann algebras and not much is said about groups. The lack of study of general square-integrable representations would not be much of a deficiency were it not for the fact that many discrete groups and Lie groups have

Received by the editors March 8, 1976.

AMS (MOS) subject classifications (1970). Primary 22D10, 22E45, 22E50; Secondary 22D25, 46L25, $22 \mathrm{E} 25$.

(')Supported by an NSF Graduate Fellowship. Final preparation of the manuscript was also supported by the Graduate Division of the University of California, Berkeley. 
non-type I regular representations which are both tractable and interesting. For instance, no infinite discrete group can have any square-integrable irreducible representations [36, Corollary 5.12], yet as we shall see, many such groups have computable square-integrable factor representations. These enter into the central decomposition of the regular representation in the same way that square-integrable irreducible representations enter into the Plancherel formula of a type I group.

The body of this paper is in four sections. $\$ 2$ contains a brief exposition of the general theory of square-integrable factor representations of locally compact groups, designed to fill at least part of the gap discussed above. The results are less complete than in the type I case because one can no longer define a "formal degree" in the usual way $\left.{ }^{2}\right)$. $\$ 2$ also contains some more specific results about almost connected and discrete groups, most notably the fact that every square-integrable factor representation of an almost connected group is traceable; some connections with the topology of the primitive ideal space of the group $C^{*}$-algebra are also included. $\$ 3$ deals with connected Lie groups, and relies heavily on recent advances made by L. Pukanszky [29]-[33] in the study of the representation theory of these groups. We give a characterization of the square-integrable factor representations of Lie groups which, when specialized to the case of type I solvable groups, verifies a conjecture of C. C. Moore and J. Wolf [24]. We also prove a sufficient condition for a primitive ideal in the group $C^{*}$-algebra of a solvable Lie group to be maximal, partially clarifying some results of [23] and [33] and relevant to unimodular groups with square-integrable factor representations. $\$ \$ 4$ and 5 deal with the more classical situation of type I groups, but we include them in this paper since the results amplify the results of $\$ 3$. \$4 concerns exponential Lie groups, to which (especially in the unimodular case) one can generalize most of the results of [24], and $\$ 5$ concerns certain $p$-adic solvable algebraic groups studied by R. Howe [18]. Our approach in $\$ 4$ is via a theorem (3.5) of $\$ 3$ and the methods of Moore and Wolf, rather than by an alternate method of M. Duflo and M. Raiis [11].

The author is indebted to Professors Marc A. Rieffel and Calvin C. Moore and to Philip Green for several helpful discussions on the subject of this paper. He particularly wishes to thank Professor Moore for the proofs of Proposition 2.18 and 4.4, Professor Michèle Vergne for the proof of Lemma 4.6, and Professor Rieffel for a careful reading of the manuscript which resulted in elimination of a number of errors and obscurities.

(2) C. C. Moore has found analogues of the formal degree and second orthogonality relations in our context, but their formulation appears rather different from the traditional form for irreducible representations. 
2. General theory of square-integrable factor representations.

2.1. Notation. Throughout this paper, $G$ will generally denote a locally compact group. We use the notations $G_{0}$ for the connected component of the identity element in $G$ and $Z(G)$, or just $Z$, for the center of $G . C^{*}(G)$ denotes the group $C^{*}$-algebra $[8,13.9 .1]$ of $G$ and $\mathscr{K}(G), \mathscr{D}(G)$ denote the convolution algebras of continuous functions with compact support and (for Lie groups) $C^{\infty}$ functions with compact support on $G$, respectively, which we often view as subalgebras of $C^{*}(G)$. Prim $(G)$ denotes the primitive ideal space of $C^{*}(G)$, which is a $T_{0}$ space in the Jacobson hull-kernel topology. A representation of $G$ always means a continuous unitary representation on a Hilbert space. We sometimes identify representations with their unitary equivalence classes. If $\sigma$ is a representation of $G$, the kernel of $\sigma$ generally means not the group-theoretic kernel of $\sigma$ but rather the kernel of the associated *-representation of $C^{*}(G)$. If $H$ is a closed subgroup of $G$ and $\sigma$ is a representation of $H, \operatorname{Ind}_{H \uparrow G} \sigma$ denotes the induced representation of $G$, in the sense of Mackey and Blattner. A projective representation of $G$ on a Hilbert space $\mathscr{T C}$ is a homomorphism from $G$ to the projective unitary group $\mathrm{PU}(\mathcal{H C})$ on $\mathcal{H}$ which is continuous when $\mathrm{PU}(\mathcal{H})$ is given the quotient topology of the strong operator topology. $\mathbf{T}$ denotes the circle group of complex numbers of modulus one. If $V$ is a finite-dimensional real vector space, $V^{*}$ denotes its real dual. And if $G$ operates on a space $X, G_{x}$ denotes the stability group of $x \in X$ in $G$.

2.2. Definitions. Next we review and extend slightly some definitions from [10] and [24]. Let $\pi$ be a representation of $G$ on a Hilbert space $\mathcal{H}$. The coefficients of $\pi$ are the functions $c_{\xi_{\eta}}(\xi, \eta \in \mathcal{H})$ on $G$ given by $c_{\xi \eta}(g)=$ $\langle\xi, \pi(g) \eta\rangle(g \in G)$. Coefficients are, of course, bounded and continuous. If $\pi$ is a factor representation, $\pi$ is said to be square-integrable in the strict sense (S.I.S.S.) if $\pi$ has at least one nonzero square-integrable coefficient. Let $Z=Z(G)$. For a factor representation $\pi, \pi(Z)$ consists of scalar multiples of the identity operator, so that $\pi \mid Z$ is a multiple of a unitary character of $Z$. In particular, the absolute value of a coefficient for $\pi$ is constant on cosets of $Z$, and may be viewed as a function on $G / Z$. We say that $\pi$ is square-integrable in the weak sense (S.I.W.S.) if for some nonzero coefficient $c_{\xi_{\eta}}$ of $\pi,\left|c_{\xi_{\eta}}\right| \in$ $L^{2}(G / Z)$. As pointed out for irreducible representations in [24], when $Z$ is compact, $\pi$ is S.I.S.S. if and only if $\pi$ is S.I.W.S., and when $Z$ is not compact, $G$ has no S.I.S.S. factor representations. If $\sigma$ is a projective representation of $G$, then (with slight abuse of notation) it still makes sense to define $\left|c_{\xi \eta}\right|(g)=$ $\langle\xi, \sigma(g) \eta\rangle \mid$ for $g \in G$, and $\left|c_{\xi \eta}\right|$ is a continuous function. We say $\sigma$ is S.I.S.S. if $\left|c_{\xi \eta}\right| \in L^{2}(G)$ for some $\xi, \eta \in \mathcal{H}$ such that $\left|c_{\xi \eta}\right| \neq 0$. S.I.W.S. representations of $G$ may always be viewed as S.I.S.S. projective representations of the group $G / Z$, and therefore the theories of S.I.W.S. and S.I.S.S. representations are essentially the same in most respects. 
2.3. Proposition. Let $G$ be a locally compact group, $\pi$ a factor representation of $G$.

(a) Then the following are equivalent:

(i) $\pi$ is S.I.S.S.

(ii) $\pi$ is quasi-equivalent to a subrepresentation of the left regular representation $\lambda$ of $G$.

(b) Let $Z$ be the center of $G$ and let $\chi$ be the element of $\hat{Z}$ of which $\pi \mid Z$ is a multiple. Then the following are equivalent:

(i) $\pi$ is S.I.W.S.

(ii) $\pi$ is quasi-equivalent to a subrepresentation of $\operatorname{Ind}_{Z \uparrow G} X$.

Proof. (a) When $G$ is separable, this is a direct consequence of [7, III. 2.7-III. 2.12]. However, even in this case, it seems preferable to give a direct proof. We essentially repeat the argument of [10, Theorem 2]. First assume (ii), and let $\sigma$ be a subrepresentation of $\lambda$ quasi-equivalent to $\pi$. The argument of [10] applies without change to show that $\sigma$ has a nonzero square-integrable coefficient. Let $<$ denote containment of representations. Since $\pi$ and $\sigma$ are quasi-equivalent, either $\pi \leqslant \sigma$ or $\sigma \leqslant \pi$ (see [8, Propositions 5.2.9 and 5.3.1 D. In the first case, by transitivity of $\leqslant, \pi \leqslant \lambda$, and we can replace $\sigma$ by $\pi$ in the reasoning above to see that (i) holds. In the second case, a nonzero square-integrable coefficient for $\sigma$ may be viewed as one such for $\pi$.

Now assume (i). As in [10], choose $\xi, \eta \in \mathcal{K}_{\pi}$ such that $c_{\xi \eta} \neq 0$ and $c_{\xi \eta} \in L^{2}(G)$, and define $D_{\eta}:\left\{\xi \in \mathcal{H}_{\pi}: c_{\xi \eta} \in L^{2}(G)\right\} \rightarrow L^{2}(G)$ by $D_{\eta}(\xi)=$ $c_{\xi \eta^{*}}$. Then $D_{\eta}$ is a closed intertwining operator between $\pi$ and $\lambda$. The nullspace $V$ of $D_{\eta}$ is closed and $\pi(G)$-invariant, and restricting to the orthogonal complement of $V$ we may assume $D_{\eta}$ is one-to-one. ( $\pi$ is quasi-equivalent to any of its subrepresentations.) Similarly we may cut down to a smaller invariant subspace and assume $D_{\eta}$ is densely defined. Then as in [10, Theorem 1], polar decomposition of the closed operator $D_{\eta}$ produces a unitary intertwining operator between $\pi$ and a subrepresentation of $\lambda$. This proves (ii).

(b) is proved in exactly the same fashion, using the explicit definition of an induced representation. It can also be deduced directly from (a), using the natural correspondence between projective representations of $G / Z$ and ordinary unitary representations of certain extensions of $G / Z$ by $T$. (This correspondence may be established using pull-backs even when $G / Z$ is not separable-for the very easy argument, see $\$ 8$ of [37].)

2.4. Proposition (The “ORThogonality Relation"). If $\pi_{1}$ and $\pi_{2}$ are non-quasi-equivalent S.I.S.S. factor representations of $G$ with square-integrable coefficients $c_{1}$ and $c_{2}$, respectively, then $c_{1} \perp c_{2}$ in $L^{2}(G)$. Similarly, if $\pi_{1}$ and $\pi_{2}$ are non-quasi-equivalent S.I.W.S. factor representations of $G$ both restricting to 
multiples of the same character $\chi$ of $Z$, and if $c_{1}$ and $c_{2}$ are coefficients of $\pi_{1}$ and $\pi_{2}$, respectively, with $\left|c_{1}\right|,\left|c_{2}\right| \in L^{2}(G / Z)$, then $c_{1} \perp c_{2}$ in the Hilbert space of Ind $_{Z \uparrow G} X$.

Proof. We prove only the first case; the second is completely analogous. Let $\mathcal{F}_{1}, \mathcal{F}_{2}$ be the cyclic left-invariant subspaces of $L^{2}(G)$ generated by $c_{1}, c_{2}$, respectively, and let $P_{1}, P_{2}$ be the corresponding orthogonal projections. If $\mathcal{G}_{1}$ and $\mathcal{F G}_{2}$ are not orthogonal, then $\lambda \mid\left(P_{1} \mathcal{F}\right)^{-}$or $\left.\lambda \mid\left(P_{2} \mathcal{T}\right)_{1}\right)^{-}$ is a nonzero factor representation of $G$ quasi-equivalent to both $\pi_{1}$ and $\pi_{2}$, a contradiction.

2.5. The next several propositions are all implicit in the work of Pukanszky [32], but we spell them out in detail in preparation for the proofs of Theorem 2.13 and Proposition 2.14. Besides, they are of considerable interest in their own right.

But first we need to recall some definitions from the theory of $C^{*}$-algebras. If $A$ is a $C^{*}$-algebra, a traced representation of $A[8,6.6 .1]$ is a pair $(\pi, \tau)$, where $\pi$ is a *-representation of $A$ and $\tau$ is a faithful normal semifinite trace on the von Neumann algebra $\pi(A)$ ", such that the intersection of $\pi(A)$ with the ideal of definition of $\tau$ is weakly dense in $\pi(A)^{\prime \prime}$. When $(\pi, \tau)$ is a traced representation of $A$, we may view $\tau$ as a trace on $A$ (rather than on $\left.\pi(A)^{\prime \prime}\right)$, and we say $\pi$ is a traceable representation. A traceable factor representation is also called a normal representation. The same terms apply, of course, to locally compact groups, since we may identify representations of $G$ and of $C^{*}(G)$.

A homogeneous representation [12] of a $C^{*}$-algebra is a ${ }^{*}$-representation every nonzero subrepresentation of which has the same kernel. Factor representations are always homogeneous.

2.6. Proposition ("Pukanszky's Lemma"). Let $G$ be a locally compact group, and let $N$ be a closed normal subgroup of $G$, such that $G / N$ is unimodular. Let $(\pi, \tau)$ be a traced representation of $N$ such that the trace $\tau$ on $C^{*}(N)$ is G-invariant. Then $\operatorname{Ind}_{N \uparrow G} \pi$ is traceable.

Proof. This is essentially proved, although nowhere stated, in [32, \$2]. ( $N$ here takes the place of Pukanszky's $L ; \pi$ takes the place of his $\int_{E}^{\oplus} \pi d \omega$.) The key to the argument is the construction of a subalgebra $\mathscr{B}$ of $C^{*}(G)$ (cf. [32, Lemma 2.1.1]), consisting of covariant functions from $G$ into $C^{*}(N)$, on which there is a natural trace. Pukanszky uses existence of a continuous cross-section from $G / N$ to $G$ on pp. 98, 103, and 105 of his paper, but this turns out to be unnecessary if we modify his proofs or the definition of $B$. When $G$ is separable (the only case we need), there exists a Borel crosssection $G / N \rightarrow G$ [1, Chapter I, Proposition 3.2], which we may use instead of a continuous cross-section wherever one is needed, provided we let 


$$
\begin{aligned}
\mathscr{B}=\{\text { bounded Borel functions } f: G & \rightarrow C^{*}(N) \text { of compact support } \\
\text { modulo } N \mid f(n x) & =R(n) f(x) \text { for all } n \in N, x \in G\} .
\end{aligned}
$$

Here $R$ denotes the right regular representation of $N$ on $C^{*}(N)$. Then if we let a superscript $s$ denote the *-operation on $C^{*}(N)$, we can show that for $f \in \mathscr{B},\|f\|_{\tau}^{2}=\int_{G / N} \tau\left(f(x)^{s} f(x)\right) d \dot{x}$ is well defined and $\|f\|_{\tau}=\left\|f^{*}\right\|_{\tau} \cdot\|\cdot\|_{\tau}$ then gives rise to the scalar product of a Hilbert algebra corresponding to a trace for $\operatorname{Ind}_{N \uparrow G} \pi$.

To handle the general case, we may revert to Pukanszky's definition of $\mathscr{B}$ (the same as above, but with "continuous" replacing "Borel"). It is then necessary to prove density of $\mathscr{B}$ in $C^{*}(G)$ and existence of enough elements $f \in \mathscr{B}$ with $\|f\|_{\tau}<\infty$. The arguments for this (using a Bruhat "approximate cross-section") are quite similar to those used by Blattner in [4]; we omit them since they are not relevant to the rest of this paper.

2.7. Corollary. Let $G$ be a locally compact group, and let $N$ be an open subgroup of finite index in $G$. Let $\sigma$ be a traceable representation of $N$. Then Ind $_{N \uparrow G} \sigma$ is traceable.

Proof. By $[8,6.6 .7]$, it is enough to show that a representation quasiequivalent to $\operatorname{Ind}_{N \uparrow G} \sigma$ is traceable. Let $\pi=\left(\operatorname{Ind}_{N \uparrow G} \sigma\right) \mid N$. Since $G / N$ is finite, $\pi$ is a direct sum of finitely many representations $g_{i} \cdot \sigma$, where $g_{i}$ runs over a set of representatives for $G / N$ in $G$. Since $g_{i} \cdot \sigma$ and $\sigma$ induce the same representation of $G$ (up to equivalence), $\operatorname{Ind}_{N \uparrow G} \pi$ is quasi-equivalent to Ind $_{N \uparrow G} \sigma$. Now if $\tau$ is a trace for $\sigma, \Sigma g_{i} \cdot \tau$ is clearly a $G$-invariant trace for $\pi$, so we need only apply the proposition above or [3, Corollary 3.3.7].

2.8. Recall that the group $G$ is said to be almost connected if $G / G_{0}$ is compact. A Lie group is almost connected if and only if it has only finitely many connected components.

LEMMA. Let $G$ be an almost connected locally compact group, and let $J \in \operatorname{Prim}(G)$. Then $J$ is the kernel of (up to quasi-equivalence) at most finitely many normal representations.

Proof. When $G$ is a connected Lie group, Pukanszky [32, Theorem 1, p. 119] proved something much stronger, namely that $J$ is the kernel of a normal representation, unique up to quasi-equivalence. We are confident that this stronger statement remains true for all almost connected groups, but here we only prove as much as we need for what follows. (The existence of a normal representation with kernel a given primitive ideal can be deduced from a new result of D. Poguntke [27], but uniqueness seems to be harder.)

Note first of all that by [22, Proposition 2.2], or by [21], it is enough to consider the case where $G$ is an almost connected Lie group. So assume $G$ is Lie, and let $\rho$ be a normal representation of $G$ with kernel $J$. By the usual 
Mackey subgroup analysis (see for instance [1, Chapter I, §10] or [37]), $\rho \mid G_{0}$ is a finite direct sum of factor representations $g_{i} \cdot \pi$ of $G_{0}$, where $g_{i}$ runs over a set of representatives for $G / G_{0}$ in $G$. Since $G_{0}$ is open and of finite index in $G$, we know that $C^{*}\left(G_{0}\right)$ is a subalgebra of $C^{*}(G)$, and that $C^{*}(G)=\Sigma g_{i}$. $C^{*}\left(G_{0}\right)$. From this and the fact that $\rho$ is traceable, it is easy to see that some $g_{i} \cdot \pi$, and hence every summand of $\rho \mid G_{0}$ (since the summands are all conjugates of each other), is traceable. By the theorem for connected Lie groups, $\pi$ is then determined uniquely up to quasi-equivalence by its kernel. But the kernels of the $g_{i} \cdot \pi$ are determined by $J$, so $\rho \mid G_{0}$ is determined by $J$ up to quasi-equivalence. Then since $\rho$ is a factor subrepresentation of $\operatorname{Ind}_{G_{0} \uparrow G}\left(\rho \mid G_{0}\right)$, which is a finite direct sum of factor representations, we see that, up to quasi-equivalence, there are only finitely many possibilities for $\rho$.

2.9. Lemma. Let $G$ be a connected, simply connected Lie group. Then $G$ can be written as a direct product $V \times G^{\prime}$, where $V$ is a vector group and $Z\left(G^{\prime}\right)_{0} \subseteq\left[G^{\prime}, G^{\prime}\right]$.

Proof. Let $g$ be the Lie algebra of $G$, let $\mathfrak{l}=[\mathfrak{g}, \mathfrak{g}]$, and let $z$ be the center of $g$. Letting $\mathfrak{b}$ be a complement to $z \cap \mathfrak{l}$ in $z$, we can choose a complement $a$ for $z+\mathfrak{l}=\mathfrak{b}+\mathfrak{l}$ in $\mathfrak{g}$ and let $\mathfrak{g}^{\prime}=\mathfrak{a}+\mathfrak{l}$. Note that $[\mathfrak{b}, \mathfrak{g}]=0$ and $\left[\mathfrak{g}^{\prime}, \mathfrak{g}^{\prime}\right] \subseteq \mathfrak{l}$ $\subseteq \mathfrak{g}^{\prime}$, so that $g$ is the Lie algebra direct sum of $\mathfrak{b}$ and $\mathfrak{g}^{\prime}$. Then if $V$ and $G^{\prime}$ are the analytic subgroups of $G$ with Lie algebras $\mathfrak{b}$ and $\mathfrak{g}^{\prime}$, we have $G=V \times G^{\prime}$ and $Z\left(G^{\prime}\right)_{0} \subseteq\left[G^{\prime}, G^{\prime}\right]$.

2.10. THEOREM. Let $G$ be a connected, simply connected Lie group, and let $D$ be a closed central subgroup of $G$. Then for any $\chi \in \hat{D}, \operatorname{Ind}_{D \uparrow G} \chi$ is the central direct integral, with respect to a Borel measure on $\operatorname{Prim}(G)$, of traceable homogeneous representations.

Proof. Write $G=V \times G^{\prime}$ as in the last lemma, and let $Z=Z(G)$, $L=[G, G]$. Let $\tilde{G}$ be as in [32], so that $\tilde{G}$ is a locally algebraic simply connected group containing $G$ and satisfying $[\tilde{G}, \tilde{G}]=L$. By $[32$, Lemmas 1.1.1 and 1.1.3], $L$ is type $I$ and regularly embedded in $\tilde{G}$. Note that Ad $L=L Z / Z$ is the commutator subgroup of the algebraic group Ad $\tilde{G}$, and is therefore automatically closed in $\operatorname{Ad} \tilde{G}$ [5, pp. 107-110], hence also in $\operatorname{Ad} G=G / Z$. So $L Z$ is closed in $G$. Now $Z /(V \times(Z \cap L))$ is discrete by Lemma 2.9, and is free abelian (of finite rank) since it is algebraically isomorphic to a subgroup of the vector group $G /(V \times L)$. So the group extension

$$
0 \rightarrow V \times(Z \cap L) \rightarrow Z \rightarrow Z /(V \times(Z \cap L)) \rightarrow 0
$$

splits and we may choose a complement $Z^{\prime}$ to $V \times(Z \cap L)$ in $Z$. Since $L Z$ is closed and $Z$ is central in $G$, we also have a topological group isomorphism $L Z \cong V \times L \times Z^{\prime}$. It follows that $L V D$ is closed in $G$ and is of the form 
$V \times L \times D^{\prime}$, with $D^{\prime}$ a complement to $V \times(D \cap L)$ in $D$. ( $D^{\prime}$ is, of course, free abelian of finite rank.)

It is convenient to now let $Q=V \times L$. Fix $\chi \in \hat{D}$. Since $D \cong(D \cap Q)$ $\times D^{\prime}$, we may write $\chi$ as $\chi_{1} \times \chi_{2}$, with $\chi_{1} \in(D \cap Q)$ and $\chi_{2} \in \hat{D}^{\prime}$. Then since $Q D \cong Q \times D^{\prime}$,

$$
\operatorname{Ind}_{D \uparrow G} \chi \cong \operatorname{Ind}_{Q D \uparrow G}\left(\left(\operatorname{Ind}_{(D \cap Q) \uparrow Q} \chi_{1}\right) \times \chi_{2}\right) .
$$

Choose a measure $\mu$ in the "semi-Plancherel" measure class of the canonical decomposition of $\operatorname{Ind}_{(D \cap Q) \uparrow Q} X_{1}$ over $\hat{Q}$. As on p. 110 of [32], we can fiber $\mu$ as $\int_{\hat{Q} / \tilde{G}} \mu_{s} d \nu(s)$, where $\mu_{s}$ is a $\hat{G}$-quasi-invariant measure on the orbit $s$. Then if $\Pi_{s}=\int_{s}^{\oplus} \pi(\zeta) d \mu_{s}(\zeta)$ with $\pi$ a measurable cross-section from $\hat{Q}$ to the concrete irreducible representations of $Q, \int \frac{\oplus}{Q} / \hat{\sigma} \Pi_{s} d \nu(s)$ is quasi-equivalent to $\operatorname{Ind}_{(D \cap Q) \uparrow Q} \chi_{1}$. Exactly as in [32, p. 110], $\sigma_{s}=\operatorname{Ind}_{Q D \uparrow G}\left(\Pi_{s} \times \chi_{2}\right)$ (which depends on $\chi$ ) is traceable for $\nu$-almost all $s \in \hat{Q} / \tilde{G}$ (here we must use Pukanszky's Lemma, Proposition 2.6 above), and $\int^{\oplus_{\sigma_{s}}} d \nu(s)$ is a central decomposition of a representation quasi-equivalent to $\operatorname{Ind}_{D \uparrow G} X$.

Using the principal results of [12], we can decompose $\int^{\oplus_{\sigma_{s}}} d v(s)$ further into a direct integral, with respect to a Borel measure on Prim $(G)$, of homogeneous representations. Since $\nu$-almost all of the $\sigma_{s}$ are traceable, and since any homogeneous representation of $G$ lives on a single $\tilde{G}$-orbit in $\hat{Q}[32$, Lemma 1.1.8], we see that almost all of the homogeneous representations in this decomposition are traceable. But again traceability is preserved under quasi-equivalence $[8,6.6 .7]$, so the conclusion of the proposition follows.

2.11. Proposition. Let $G$ be an almost connected Lie group, and let $\lambda_{G}$ be the left regular representation of $G$. Then $\lambda_{G}$ is the central direct integral, with respect to a Borel measure on $\operatorname{Prim}(G)$, of traceable homogeneous representations. In particular, any homogeneous subrepresentation of $\lambda_{G}$ is traceable.

Proof. Let $H$ be the universal covering group of the connected group $G_{0}$, and let $D$ be the kernel of the covering map $H \rightarrow G_{0}$. By the last theorem, $\operatorname{Ind}_{D \uparrow H} 1$ is the central direct integral (over Prim $(H)$ ) of traceable homogeneous representations. But as a $C^{*}$-algebra representation, $\operatorname{Ind}_{D \uparrow H} 1$ is just $\lambda_{G_{0}}$ lifted from $C^{*}\left(G_{0}\right)$ to $C^{*}(H)$. So $\lambda_{G_{0}}$ is the central direct integral of traceable homogeneous representations, and clearly we can base this direct integral on $\operatorname{Prim}\left(G_{0}\right)$. Say $\lambda_{G_{0}} \cong \int_{\operatorname{Prim}\left(G_{0}\right)}^{\oplus}(J) d \mu(J)$, where $\mu$ is a Borel measure and $\pi(J)$ is a traceable homogeneous representation with kernel $J$. Then

$$
\lambda_{G} \cong \int_{\operatorname{Prim}\left(G_{0}\right)}^{\oplus} \operatorname{Ind}_{G_{0} \uparrow G} \pi(J) d \mu(J) .
$$

By 2.7 above, each representation in the integrand is traceable and, therefore, further decomposes into homogeneous traceable representations of $G$. Now 
we can regroup the integrands (so as to base the direct integral on Prim $(G)$ ), and using the fact that a primitive ideal of $C^{*}(G)$ can lie over only finitely many primitive ideals of $C^{*}\left(G_{0}\right)$, we obtain the desired decomposition of $\lambda_{G}$.

2.12. The following result strengthens slightly a theorem of J. Dixmier [9]. One would actually like to prove that the regular representation of any almost connected locally compact group is traceable, but at present this seems to be known only for unimodular groups (for which the proof is trivial) and for solvable Lie groups (for which the result is Theorem 4, p. 593 of [29]-the proof is quite difficult).

THEOREM. Let $G$ be any almost connected locally compact group, and let $\lambda_{G}$ be its left regular representation. Then the von Neumann algebra generated by $\lambda_{G}$ is semifinite; furthermore, any homogeneous subrepresentation of $\lambda_{G}$ is traceable and is the direct sum of finitely many normal representations. If $G$ is separable, $\lambda_{G}$ is the central direct integral, with respect to a Borel measure on $\operatorname{Prim}(G)$, of traceable homogeneous representations.

Proof. Proceeding as in the proof of [9, Lemme 5.3], we can fix a compact normal subgroup $K$ of $G$ such that $G / K$ is Lie. Then $\lambda_{G}$ is a discrete direct sum of representations Ind $_{K \uparrow G} \rho$, where $\rho \in \hat{K}$. As in Dixmier's proof, such a representation is lifted from a subrepresentation of the regular representation of an almost connected Lie group, to which we can apply the last proposition. The desired direct integral decomposition of $\lambda_{G}$ in the separable case, and the semifiniteness of $\lambda_{\sigma}$ in the general case, are immediate. As far as the statement on homogeneous subrepresentations of $\lambda_{G}$ is concerned, we note that by [22, Proposition 2.2] or by [21], such a representation must "live" on a Lie quotient of $G$, where it then corresponds to a subrepresentation of the regular representation by the above analysis. Hence a homogeneous subrepresentation $\pi$ of $\lambda_{G}$ is traceable, again by 2.11 . Since $\pi$ is lifted from a separable quotient of $C^{*}(G)$, we can decompose $\pi$ as in [8, Théorème 8.8.2] into a central direct integral of normal representations. Since $\pi$ is homogeneous, almost all of these have the same kernel as $\pi$. By Lemma 2.8 , the integrated representations (aside from a null set, which can be discarded) then belong to only finitely many quasi-equivalence classes, and so $\pi$ is a finite sum of normal representations.

2.13. THEOREM. Let $G$ be an almost connected locally compact group. Then any S.I.W.S. factor representation of $G$ is normal.

Proof. Let $Z$ be the center of $G$, and let $\pi$ be an S.I.W.S. factor representation of $G$. By the remarks in (b) of the proof of 2.3 above, $\pi$ may be viewed as an S.I.S.S. representation of some extension $H$ of $G / Z$ by $T$. Then $H$ is almost connected, and $\pi$ is a homogeneous subrepresentation of $\lambda_{H}$, so we can apply 2.12. (One could avoid passing from $G$ to $H$ if 2.11 and 2.12 
were generalized to deal with representations induced from characters of central subgroups. The modifications in the proofs needed for this are strictly a matter of extra bookkeeping.)

2.14. Proposition. Let $G$ be a separable locally compact group which is either (i) type I or (ii) almost connected. Then any open point in $\operatorname{Prim}_{r}(G)=$ $\operatorname{Prim}\left(C_{r}^{*}(G)\right)$ is the kernel of an S.I.S.S. normal representation of $G$.

Proof. This is a direct generalization of [8, 18.9.1.a]. By an application of the main results of [12], the central decomposition of the left regular representation $\lambda$ of $G$ defines a unique "Plancherel" measure class in Prim $(G)$ with support $\operatorname{Prim}_{r}(G)$. An open point in $\operatorname{Prim}_{r}(G)$ cannot be a null set with respect to this measure class, and so $\lambda$ has a homogeneous subrepresentation with this primitive ideal as kernel. When $G$ is type $I$, any homogeneous representation is a multiple of a traceable irreducible representation, and thus the result follows in Case (i). In Case (ii), we apply Theorem 2.12.

2.15. Definition. Let $\pi$ be a factor representation of $G$. As in [10], $\pi$ is said to be integrable if it has at least one nonzero integrable (and $a$ fortiori square-integrable) coefficient. Of course one could also define integrable representations in the weak sense, but we shall have no use for such a concept.

2.16. LEMMA. Let $\pi$ be an integrable factor representation of $G$, let $\left\{\sigma_{\alpha}\right\}$ be a family of pairwise non-quasi-equivalent S.I.S.S. factor representations of $G$, and suppose that $\pi$ is weakly contained in $\left\{\sigma_{\alpha}\right\}$. Then $\pi$ is quasi-equivalent to some $\boldsymbol{\sigma}_{\boldsymbol{\alpha}} \cdot$

Proof. First we replace each $\sigma_{\alpha}$ if necessary by a quasi-equivalent subrepresentation of the left regular representation $\lambda$ of $G$. Then we form $\sigma=\bigoplus \sigma_{\alpha}$, which is a subrepresentation of $\lambda$. Let $c_{1}$ be a nonzero integrable coefficient of $\pi$. By [10, Theorem 4] (the proof does not use irreducibility of $\pi$ at this point), there exists a coefficient $c_{2}$ of $\sigma$ with $\int_{G} c_{1}(g) \overline{c_{2}(g)} d g \neq 0$. Suppose $c_{2}=c_{\zeta \eta}$ with $\xi, \eta \in L^{2}(G)$. Approximating $\eta$ by elements of $\mathscr{K}(G)$, we can find (as in the proof of [10, Theorem 2]) a nonzero coefficient $c_{3}$ of $\sigma$ with $c_{3} \in L^{2}(G)$ and $\left\langle c_{1}, c_{3}\right\rangle \neq 0$. Since $\sigma$ is the disjoint direct sum of the $\sigma_{\alpha}$ 's, $c_{3}$ is a sum of coefficients of the various $\sigma_{\alpha}$ 's. Hence there exists a nonzero coefficient $c_{4}$ of some $\sigma_{\alpha}$ with $c_{4} \in L^{2}(G)$ and $\left\langle c_{1}, c_{4}\right\rangle \neq 0$. The result now follows from Proposition 2.4 (orthogonality of coefficients).

2.17. Proposition. Let $A$ be the set of quasi-equivalence classes of integrable factor representations of $G$. Then in the topology of weak containment (the Fell topology or the pull-back to $A$ of the hull-kernel topology on the set of prime ideals of $C^{*}(G)$ which are kernels of representations from $\left.A\right), A$ is discrete. In 
particular, if $G$ is separable, $A$ defines a subset of $\operatorname{Prim}(G)$ which is discrete in the relative topology.

Proof. The first statement is immediate from Lemma 2.16. The second follows since the kernel of a factor representation of a separable group is a primitive ideal of the group $C^{*}$-algebra.

2.18. Suppose now that $G$ is discrete. Then the center $\mathscr{Z}$ of the von Neumann algebra generated by the left regular representation $\lambda$ of $G$ can be computed, at least in principle (see [39], for instance), and the S.I.S.S. factor representations of $G$ correspond exactly to the atoms of $\mathscr{Z}$. In particular, we have the following result.

Proposition. Let $G$ be a discrete group with $F C$-subgroup $F$. (By definition, $F$ is the group of elements of $G$ with finite conjugacy classes.) If $F$ is finitely generated, then either $F$ is infinite and $G$ has no S.I.S.S. factor representations, or else $F$ is finite and $\lambda$ is a direct sum of finitely many non-quasi-equivalent S.I.S.S. (in fact integrable) factor representations.

Proof. As noted in say [39], elements of $\mathscr{Z}$ may be viewed as class functions in $L^{2}(G)$, necessarily supported on $F$. So certainly finiteness of $F$ implies that $\mathscr{Z}$ is finite dimensional and atomic. It is also easy to see that, in this case, the central summands of $\lambda$ are integrable.

We now examine what happens when $F$ is infinite. Let $g_{1}, \ldots, g_{n}$ be generators for $F$, and let $Z_{F}\left(g_{i}\right)$ and $Z_{G}\left(g_{i}\right)$ be the centralizers of $g_{i}$ in $F$ and $G$, respectively. Let $F_{1}=\cap_{1<i<n} Z_{F}\left(g_{i}\right), G_{1}=\cap_{1<i<n} Z_{G}\left(g_{i}\right)$. We have $[F$ : $\left.Z_{F}\left(g_{i}\right)\right]<\left[G: Z_{G}\left(g_{i}\right)\right]<\infty$ for each $i$, so that $\left[G: G_{1}\right]<\infty,\left[F: F_{1}\right]<\infty$. Now $F_{1}=F \cap G_{1}$ is central in $G_{1}$, and hence if $F_{1}$ is infinite, $G_{1}$ has no S.I.S.S. factor representations. Since the regular representation of $G$ is induced from that of $G_{1}$, and since $\left[G: G_{1}\right]<\infty$, this implies the same for $G$. So it is enough to prove that $F_{1}$ is infinite whenever $F$ is infinite. But this is clear since $\left[F: F_{1}\right]<\infty$.

2.19. Further conditions for discrete groups to have S.I.S.S. factor representations have been found by Schlichting [38]. One interesting result of his (the corollary to his Lemma 4 ) is that if $G$ has an S.I.S.S. factor representation, then $F$ is necessarily a torsion group, and in fact, $F$ is locally finite.

There is an analogue of Proposition 2.18 for S.I.W.S. factor representations of discrete groups with infinite center $Z$, which may be obtained by viewing such representations as projective representations of $G / Z$ and considering in place of $F$ the set of $\omega$-regular elements [20] of $G / Z$, for appropriate cocycles $\omega \in Z^{2}(G / Z, T)$.

2.20. One might hope that in addition to Propositions 2.14 and 2.17, one could formulate results about square-integrable factor representations generalizing other results such as [8,18.9.1.b] or [10, Corollary to Theorem 4] 
relating the square-integrable irreducible representations of $G$ to the topology of $\hat{G}$. As we shall see later, one interesting property remains valid for unimodular connected solvable Lie groups not necessarily of type I; however, most of the obvious conjectures fail. To begin with, one can make no general assertions about the von Neumann-Murray type of a square-integrable factor representation, as can be seen from known examples of groups with primary regular representations of types $\mathrm{I}_{\infty}, \mathrm{II}_{1}, \mathrm{II}_{\infty}$, and $\mathrm{III}_{\lambda}$ for varying $\lambda \in[0,1]$. (Consider, respectively, the affine group of the real line, a discrete group with infinite conjugacy classes, the direct product of the two preceding examples, and the adelic affine groups of [3]. Many other examples are also known.) Secondly, if $G$ is separable and $\pi$ is an S.I.S.S. factor representation of $G$, the kernel of $\pi$ need not be a closed, an open, or even a locally closed point in $\operatorname{Prim}_{r}(G)$, even if $\pi$ is integrable and $G$ is unimodular. A counterexample is provided by the semidirect product of the additive group of dyadic rationals and the multiplicative group of the integral powers of 2 , analyzed by Guichardet in [17]. This group has a primary regular representation but a highly pathological primitive ideal space.

\section{The case of connected Lie groups.}

3.1. We turn now to the specific analysis of the S.I.W.S. factor representations of connected Lie groups, following the pattern of [24]. We consider only simply connected groups, since any S.I.W.S. representation of a connected Lie group lifts to such a representation of the universal covering group. Indeed, suppose $\pi$ is an S.I.W.S. factor representation of the connected Lie group $G$. Let $H$ be the universal covering group of $G$ and let $p: H \rightarrow G$ be the canonical map. Then $\pi \circ p$ is a factor representation of $H$ with coefficients square-integrable modulo $p^{-1}\left(Z(G)\right.$ ); but $p^{-1}(Z(G))=Z(H)$ since the canonical map $q: H \rightarrow \operatorname{Ad} H$ factors through $G$ and $Z(H)=\operatorname{ker} q$.

Therefore let $G$ be a connected, simply connected Lie group with center $Z$ and commutator subgroup $L=[G, G]$. We always denote Lie groups by capital Latin letters and their Lie algebras by the corresponding lower case Gothic letters. By Lemma 2.9, we can write $G=V \times G^{\prime}$, where $V$ is a vector group and $Z\left(G^{\prime}\right)_{0} \subseteq\left[G^{\prime}, G^{\prime}\right]$. Thus factor representations of $G$ split as products of characters of $V$ and of factor representations of $G^{\prime}$, so that to study S.I.W.S. representations of $G$, we may as well replace $G$ by $G^{\prime}$ and assume that $Z_{0} \subseteq L$.

We let $Z_{1}=Z \cap L$ and argue according to the proof of Theorem 2.10, with $Z$ replacing $D$, and with a subgroup $Z_{2} \cong Z / Z_{1}$ of $Z$ replacing $D^{\prime}$. Then given a character $\chi \times \gamma$ of $Z \cong Z_{1} \times Z_{2}\left(\chi \in \hat{Z}_{1}, \gamma \in \hat{Z}_{2}\right)$, we denote by $\mu^{x}$ (this is the $\mu$ of 2.10) a measure in the class of the canonical decomposition of $\operatorname{Ind}_{z_{1} \uparrow L} \chi$ over $\hat{L}$. We generally adhere to the notations of [32], except that for consistency we denote the Lie algebra of $L$ by $\mathfrak{l}$. For the 
reader's convenience, we briefly recall some of these notations. Given $E \in$ $\hat{L} / \tilde{G}$, there exists a closed subgroup $K=K(E)$ of $G$ [32, Lemma 1.1.4] such that if $\pi \in E, \pi$ extends to a representation $\rho$ of $K$ and $\operatorname{Ind}_{K \uparrow G} \rho$ is a factor representation. It is evident from the construction of $K$ (see [29, Lemma I.3.5, p. 481]) that $Z \subseteq K$. We define $F=F(E)$ to be the subset of $\hat{K}$ consisting of all extensions of elements of $E$; then $F$ is homeomorphic to a connected abelian Lie group, since [32, Lemma 1.1.5] it is Hausdorff and the connected abelian Lie group $(\tilde{G} / K) \times(K / L)$ operates transitively on it. $F / \Sigma[32$, pp. 87-89] denotes the space of $G$-orbit closures in $F$ ( $G$ acts by conjugation on representations of the normal subgroup $K$ ). By [32, Proposition 1], $F / \Sigma$ parameterizes the elements of $\operatorname{Prim}(G)$ lying over $E$.

Note that by [13, Lemma 5.1], the operation of restricting representations from $K$ to $Z_{2}$ defines a continuous map $r$ from $F$ to $\hat{Z}_{2}$, which is surjective since $\hat{Z}_{2}$ is a quotient group of $(K / L)$. Also, every $G$-orbit lies over a unique point in $\hat{Z}_{2}$ and, by continuity of $r$, the same is true for every $\Sigma$-orbit. So $r$ induces a natural map $r^{\prime}$ from $F / \Sigma$ onto $\hat{Z}_{2}$.

3.2. THEOREM. Let $G$ be a connected and simply connected Lie group with $Z_{0} \subseteq L$ and all notation as above. Then in terms of the description of the normal representations of $G$ given in [32], the S.I.W.S. factor representations of $G$ are (up to quasi-equivalence) exactly the normal representations of $G$ lying over $\tilde{G}$-orbits $E$ in $\hat{L}$ for which

(i) $\mu^{x}(E)>0$ for some $\chi \in \hat{Z}_{1}$, and

(ii) the natural map $r^{\prime}$ from $F(E) / \Sigma$ onto $\hat{Z}_{2}$ is a covering map.

Proof. The fact that every S.I.W.S. factor representation of $G$ is normal is just a special case of Theorem 2.12. Fix $\chi \in \hat{Z}_{1}$ and $\gamma \in \hat{Z}_{2}$. For $E \in \hat{L} / \tilde{G}$, define $\sigma_{E, \gamma}=\sigma_{E}$ and $\Pi_{E}$ to be as in the proof of 2.10 ( $E$ replacing $s, \gamma$ replacing $\left.\chi_{2}\right)$. The central decomposition of $\operatorname{Ind}_{Z \uparrow G}(\chi \times \gamma)$ will contain a discrete summand if and only if (a) $\mu^{\chi}(E)>0$ for some $E \in \hat{L} / \tilde{G}$, and (b) the corresponding $\sigma_{E}$ has a factor subrepresentation. In particular, the necessity of (i) in the theorem is obvious.

Fix $E$ for the moment, and recall that $Z \subseteq K=K(E)$. By induction in stages, $\sigma_{E}$ is induced from the representation $T=\operatorname{Ind}_{L Z \uparrow K}\left(\Pi_{E} \times \gamma\right)$, and $T$ is clearly supported on $r^{-1}(\gamma) \subseteq F=F(E) \subseteq \hat{K}$. We may suppose that $\tilde{G}$ was chosen so that $Z \subseteq Z(\tilde{G})$; then the action of $\tilde{G}$ on $\hat{K}$ preserves $r^{-1}(\gamma)$, and $(\tilde{G} / K) \times(K / L Z)^{n}$ acts transitively on $r^{-1}(\gamma)$. Therefore $r^{-1}(\gamma)$, like $F$, is homeomorphic to an abelian Lie group (in this case not necessarily connected), and carries a measure $m$ (Haar measure of this group) invariant under $\tilde{G}$. From the construction of $\Pi_{E}$ and definition of $T$, it is clear that $T$ is quasi-equivalent to the direct integral, with respect to $m$, of all representations in $r^{-1}(\gamma)$. 
From Pukanszky's parameterization of $\operatorname{Prim}(G)$, we see that the central decomposition of $\sigma_{E}$ is obtained by inducing up to $G$ the disintegration of $T$ over $F / \Sigma$. From our description of $T$ as a direct integral, we then see that $\sigma_{E}$ will have a factor subrepresentation if and only if some $\Sigma$-orbit in $r^{-1}(\gamma)$ has positive $m$-measure. But since $r^{-1}(\gamma)$ is homeomorphic to an abelian Lie group in which $\Sigma$-orbits correspond to cosets of some closed subgroup (the closure of the image of $G / K)$, this happens if and only if $r^{-1}(\gamma) / \Sigma$ is discrete. Finally, $r^{-1}(\gamma) / \Sigma$ is discrete if and only if every fiber of $F / \Sigma$ over a point of $\hat{Z}_{2}$ is discrete (since all fibers are readily seen to be homeomorphic to each other), which happens if and only if $r^{\prime}$ is a covering map.

3.3. REMARKs. The theorem above, although it applies to all connected Lie groups (see the comments at the beginning of 3.1), gives essentially no information in the semisimple case, for when $G=[G, G]$, the theorem reduces to Proposition 2.3. We should also note that conditions (i) and (ii) are both necessary, since (ii) is trivial in the semisimple case and (i) is trivial when $G$ is nilpotent with $[G, G]=Z$. (There exist two-step nilpotent groups with no S.I.W.S. representations, such as the group with Lie algebra $g=\mathbf{R} e_{1}+$ $\mathbf{R} e_{2}+\mathbf{R} e_{3}+\mathbf{R} e_{4}+\mathbf{R} e_{5}$, where $\left[e_{1}, e_{2}\right]=e_{4},\left[e_{1}, e_{3}\right]=e_{5}$, and other brackets vanish.)

Now suppose that in addition to the assumptions above, $G$ is also solvable. Then $L$ is nilpotent, hence exponential, and $Z_{1}=Z_{0}$. We can use the description of Prim $(G)$ in terms of "generalized orbits" of the coadjoint representation to give an alternate version of Theorem 3.2 closer in form to the results of [24]. Recall $([32, \S 6]$ and [31, III]) that given $E \in \hat{L} / \tilde{G}$, we can consider the inverse image $O$ of $E$ in $I^{*}$ (the dual of the Lie algebra of $L$ ) under the Kirillov isomorphism $I^{*} / L \rightarrow \hat{L}$, and then let $\Omega=i^{*-1}(O)$, where $i^{*}: g^{*} \rightarrow I^{*}$ is the restriction map. For each $g \in g^{*}$, there is a corresponding character $\chi_{g}$ of $\left(G_{g}\right)_{0}$. The quotient group $G_{g} /\left(G_{g}\right)_{0}$ is free abelian of finite rank [29, Corollary I.4.1, p. 492], and $\chi_{g}$ extends to a subgroup $\bar{G}_{g}$ of $G_{g}$, called the "reduced stabilizer" of $g$. Let $\bar{G}_{g}=\left\{\chi \in \bar{G}_{g}^{\wedge}: \chi \mid\left(G_{g}\right)_{0}=\chi_{g}\right\}$. Then $\mathscr{B}(\Omega)=\cup_{g \in \Omega} \overline{\bar{G}}_{g}$ is a trivial torus bundle over $\Omega$, and the space of $G$-orbit closures in $\mathscr{B}(\Omega), \mathscr{B}(\Omega) / \mathcal{S}$, is canonically homeomorphic to $F(E) / \Sigma$. We have the following reformulation of Theorem 3.2.

3.4. Theorem. Assume the hypotheses of Theorem 3.2 and also that $G$ is solvable. With notation as above, the S.I.W.S. factor representations of $G$ are (up to quasi-equivalence) exactly the normal representations of $G$ lying over sets $\Omega=i^{*-1}(O)\left(O\right.$ a $\tilde{G}$-orbit in $\mathrm{I}^{*}$ as above $)$ in $\mathrm{g}^{*}$ such that

(i) $\operatorname{dim}(\mathfrak{l} / z)=\operatorname{dim}\left(\tilde{\mathbb{g}} / \tilde{g}_{f}\right)$ for any $f \in O=i^{*}(\Omega)$, where

$$
\tilde{\mathrm{g}}_{f}=\{x \in \tilde{\mathrm{g}}: f([x, \mathfrak{l}])=0\},
$$

and 
(ii) $\mathscr{B}(\Omega) / S$ is a covering space of $\left(Z / Z_{0}\right)$.

Proof. (ii) is obviously equivalent to (ii) in Theorem 3.2, since the natural map from $B(\Omega)$ onto $F$ is continuous and open [31, Lemma 6, p. 91]. Fixing $f \in O, O$ is a locally closed submanifold of $I^{*}$ contained in the hyperplane $f+z^{\perp}$, where $z^{\perp}$ is the annihilator in $I^{*}$ of the Lie algebra of $Z_{0}$. (This is because $z$ is central in $\tilde{g}$ as well as in g.) It is easily seen from [24] or from the Kirillov character formula that condition (i) of Theorem 3.2 is met if and only if $O$ has positive Lebesgue measure in $f+z^{\perp}$, which can happen only if $O$ meets $f+z^{\perp}$ in some open set. This in turn will happen if and only if $l / z$ and $\tilde{\mathrm{g}} / \tilde{\mathrm{g}}_{f}$ have the same dimension.

3.5. The situation is considerably simpler when $G$ is type $I$, for then $G_{g}=\bar{G}_{g}$, the orbits of $G$ on $g^{*}$ are locally closed, and $\mathscr{B}(\Omega) / \widetilde{\Phi} \cong(\Omega / G) \times$ $\left(G_{g} /\left(G_{g}\right)_{0}\right)^{\wedge}\left(\Omega\right.$ the inverse image in $g^{*}$ of a $\tilde{G}$-orbit in $I^{*}$ and $\left.g \in \Omega\right)$. The following theorem confirms a conjecture in [24]:

THEOREM $\left({ }^{3}\right)$. Suppose $G$ is a connected, simply connected, type I solvable Lie group with center Z. Then the S.I.W.S. irreducible representations of $G$ are exactly the irreducible representations obtained via the Auslander-Kostant algorithm from orbits $G g, g \in \mathrm{g}^{*}$, such that $G_{g} / Z$ is compact.

Proof. It is clearly enough to prove this when $Z_{0} \subseteq L$, so that Theorem 3.4 applies. Let $f \in O, O$ a $G$-orbit in $\mathfrak{I}^{*}$, and let $g \in \Omega=i^{*-1}(O)$. The dimension of $O$ is always $<\operatorname{dim}(\mathfrak{l} / z)$, with equality if and only if (i) of 3.4 holds. Hence

$$
\operatorname{dim} \Omega \leqslant \operatorname{dim}(\mathfrak{l} / \mathfrak{z})+\operatorname{dim}(\mathfrak{g} / \mathfrak{l})=\operatorname{dim}(\mathfrak{g} / \mathfrak{z})
$$

and

$$
\operatorname{dim}(\Omega / G)<\operatorname{dim}(\mathrm{g} / \mathfrak{\jmath})-\operatorname{dim}\left(\mathrm{g} / \mathfrak{g}_{g}\right)=\operatorname{dim}\left(\mathfrak{g}_{g} / \mathfrak{z}\right)=\operatorname{dim}\left(\left(G_{g}\right)_{0} / Z_{0}\right),
$$

with equality if and only if (i) of 3.4 holds. But

$$
\operatorname{dim}(\mathscr{B}(\Omega) / S)=\operatorname{dim}(\Omega / G) \times \operatorname{dim}\left(G_{g} /\left(G_{g}\right)_{0}\right)^{\wedge}>\operatorname{dim}\left(Z / Z_{0}\right)^{\wedge},
$$

with equality if and only if (ii) of 3.4 holds. So

$$
\begin{aligned}
\operatorname{dim}\left(\left(G_{g}\right)_{0} / Z_{0}\right)+\operatorname{dim}\left(G_{g} /\left(G_{g}\right)_{0}\right)^{\wedge} \geqslant \operatorname{dim}\left(Z / Z_{0}\right)^{\wedge} \\
=\operatorname{dim}\left(Z \cap\left(G_{g}\right)_{0} / Z_{0}\right)^{\wedge}+\operatorname{dim}\left(Z / Z \cap\left(G_{g}\right)_{0}\right)^{\wedge},
\end{aligned}
$$

with equality exactly when $g$ gives rise to an S.I.W.S. representation. Note that $Z \subseteq G_{g}$, and that

$\left({ }^{3}\right)$ Since this was written, the author has learned that the same result was obtained more or less simultaneously (via quite a different method) by J. Y. Charbonnel in his These $3^{\text {me }}$ Cycle, Paris. 


$$
\begin{aligned}
\operatorname{dim}\left(Z \cap\left(G_{g}\right)_{0} / Z_{0}\right)^{\wedge}= & \operatorname{rank}\left(Z \cap\left(G_{g}\right)_{0} / Z_{0}\right) \leqslant \operatorname{dim}\left(\left(G_{g}\right)_{0} / Z_{0}\right), \\
\operatorname{dim}\left(Z / Z \cap\left(G_{g}\right)_{0}\right)^{\wedge} & =\operatorname{rank}\left(Z\left(G_{g}\right)_{0} /\left(G_{g}\right)_{0}\right) \\
& \leqslant \operatorname{rank}\left(G_{g} /\left(G_{g}\right)_{0}\right)=\operatorname{dim}\left(G_{g} /\left(G_{g}\right)_{0}\right)^{\wedge} .
\end{aligned}
$$

So equality above holds exactly when $\operatorname{rank}\left(Z \cap\left(G_{g}\right)_{0} / Z_{0}\right)=\operatorname{dim}\left(\left(G_{g}\right)_{0} / Z_{0}\right)$ and $\operatorname{rank}\left(Z\left(G_{g}\right)_{0} /\left(G_{g}\right)_{0}\right)=\operatorname{rank}\left(G_{g} /\left(G_{g}\right)_{0}\right)$, or when $\left(G_{g}\right)_{0} /\left(Z \cap\left(G_{g}\right)_{0}\right) \cong$ $Z\left(G_{g}\right)_{0} / Z$ is compact and $G_{g} / Z\left(G_{g}\right)_{0}$ is finite, or equivalently, when $G_{g} / Z$ is compact.

3.6. REMARK. It is rather curious that the proof of Theorem 3.5 should make use of $\tilde{G}$, which was only introduced by Pukanszky in order to deal with non-type I groups, but the problem is that even if $G$ has locally closed orbits in $\mathrm{g}^{*}$, it may not have locally closed orbits in $\mathfrak{I}^{*}$. (See, for instance, the example on pp. 86-90 of [6].)

3.7. The last theorem of this section, although it provides some information on square-integrable factor representations of unimodular solvable Lie groups, was partially motivated by other considerations. Suppose $A$ is a $C^{*}$-algebra and $\pi$ is a normal *-representation of $A$, with $\pi(A)^{\prime \prime}$ a semifinite factor on a separable Hilbert space. Pukanszky in [33] has defined $\pi$ to be GCCR, "generalized completely continuous," if $\pi(A) \subseteq B$, where $B$ is the norm closure of the generalized trace class, or generalized Hilbert-Schmidt class, operators in the factor $\pi(A)^{\prime \prime}$. When $\pi$ is an irreducible representation on the Hilbert space $\mathcal{H}, B$ is the algebra of compact operators on $\mathcal{H}$, so that $\pi$ is GCCR if and only if it is CCR in the usual sense, which is the case if and only if ker $\pi$ is a maximal ideal of $A$. In general, if ker $\pi$ is maximal, then $\pi$ must be GCCR if it is normal (since $\pi^{-1}(B)$ is an ideal of $A$ containing ker $\pi$ ), but not conversely. (Any finite representation is GCCR, but the kernel of a finite representation of a general $C^{*}$-algebra need not be maximalconsider the $C^{*}$-algebra of a discrete solvable group with infinite conjugacy classes. The regular representation is then finite and primary, and weakly contains the trivial one-dimensional representation.) However by [33, Theorem 1], a connected Lie group has a $T_{1}$ primitive ideal space if and only if all its normal representations are GCCR. This suggests that perhaps the kernel of a GCCR representation of a connected Lie group is always a maximal ideal of the group $C^{*}$-algebra. The following proves this in a special case.

3.8. THEOREM. Let $G$ be a connected, simply connected solvable Lie group, and let $\sigma$ be a normal representation of $G$ such that for all $f \in \mathscr{D}(G), \sigma(f)$ is a generalized Hilbert-Schmidt operator in the semifinite factor $\sigma(G)^{\prime \prime}$. (It would be the same to require that $\sigma(f)$ be of generalized trace class for all $f \in$ D(G)-see [2, Proposition IX. 3.2.2, p. 250].) Then ker $\sigma$ is a closed point of $\operatorname{Prim}(G)\left(\right.$ i.e., a maximal ideal of $C^{*}(G)$ ). 
Proof. Let $J=\operatorname{ker} \sigma$ and let $\tau$ be the trace associated with $\sigma$. By Pukanszky's character formula [32, Theorem 2, p. 131], applied to the subalgebra $\mathscr{D}(G)$ of $\mathscr{B}(\mathscr{B}$ as in 2.6 or [32]) we have

$$
\tau(f)=\int_{\mathscr{Q}(J)} \operatorname{Tr}(\rho(f \mid K)) d \mu(\rho) \text { for } f \in \mathscr{D}(G)^{+},
$$

where $\mu$ is an appropriately normalized $G$-invariant Radon measure on $\mathbb{Q}=\mathscr{Q}(J)$ and the superscript ${ }^{+}$denotes the cone of functions of positive type. Hence by the hypothesis, the $[0, \infty]$-valued function $\rho \mapsto \operatorname{Tr}(\rho(f \mid K))$ belongs to $L^{1}(Q, \mu)$ for all $f \in \mathscr{D}(G)^{+}$. Since $K_{0}$ is an open subgroup of $K$ and thus $\mathscr{D}\left(K_{0}\right)$ is a subalgebra of $\mathscr{D}(K)$, we also know that $\rho \mapsto \operatorname{Tr}(\rho(f))$ belongs to $L^{1}(Q, \mu)$ for all $f \in \mathscr{D}\left(K_{0}\right)^{+}$.

Now recall that $F=F(J)$ is homeomorphic to a homogeneous space of $(\tilde{G} / K) \times\left(K_{0} / L\right)^{\wedge} \times\left(K / K_{0}\right)^{\wedge}$ and that $E=E(J)$ is homeomorphic to a homogeneous space of $\tilde{G} / L$. The set $\mathbb{Q}$ is the closure of a $G$-orbit in $F$ and hence also homeomorphic to a connected abelian Lie group $B$; under this identification, $\mu$ is just Haar measure on $B$. Let $\mathbb{Q}_{0}$ be the image of $\mathbb{Q}$ in $\hat{K}_{0}$ under the restriction map. This space is again homeomorphic to a connected abelian Lie group $B_{0}$, and one can easily see that $B \cong B_{0} \times T$, where $T$ is a torus. Under this decomposition, $d \mu \cong d \nu \otimes d t$, where $\nu$ is a $\tilde{G}$-invariant measure on $\mathbb{Q}_{0}$ (Haar measure on $B_{0}$ ) and $d t$ is Haar measure on $T$, which we normalize to have mass 1 . For $f \in \mathscr{D}\left(K_{0}\right)^{+}$and $\rho \in \mathbb{Q}, \rho(f)$ clearly depends only on the restriction $\rho_{0}$ of $\rho$ to $K_{0}$, so that

$$
\int_{\mathscr{Q}} \operatorname{Tr}(\rho(f)) d \mu(\rho)=\int_{T} \int_{\mathbb{Q}_{0}} \operatorname{Tr}\left(\rho_{0}(f)\right) d \nu\left(\rho_{0}\right) d t=\int_{\mathbb{Q}_{0}} \operatorname{Tr}\left(\rho_{0}(f)\right) d \nu\left(\rho_{0}\right)
$$

and thus $\rho_{0} \mapsto \operatorname{Tr}\left(\rho_{0}(f)\right)$ belongs to $L^{1}\left(\mathscr{Q}_{0}, \nu\right)$.

Our objective is to show that $\mathbb{Q}_{0}$ is closed in $\hat{K}_{0}$, using an idea from the proof of [31, Lemma 31]. This will be fairly easy once we know that the functions $\rho \mapsto \operatorname{Tr}\left(\rho(f)\right.$ ) on $\mathbb{C}_{0}$ (we drop the subscripts on the $\rho$ 's for convenience) vanish at infinity, but establishing this regularity property seems to require considerable (disagreeably technical) preparation. Note that $\mathbb{Q}_{0} \subseteq$ $F_{0}$, where $F_{0}$ is the image of $F$ in $\hat{K}_{0}$, and that the group $M=\left(\tilde{G} / K_{0}\right) \times$ $\left(K_{0} / L\right)^{\wedge}$ operates transitively on $F_{0}$. Let $\rho \in \mathbb{Q}_{0}$. Then $\mathbb{Q}_{0}$ is the closure in $F_{0}$ of the $G$-orbit $G \rho$, hence is contained in the closure of the $\tilde{G}$-orbit of $\rho$. We begin by showing that the orbits of $\tilde{G}$ on $F_{0}$ are closed (in the relative topology), and hence that $\mathbb{Q}_{0} \subseteq \tilde{G}$. Since $\tilde{G}$ operates on $F_{0}$ as a connected subgroup of $M$, it suffices to show that the action of $\tilde{G}$ on $F_{0}$ is countably separated. The proof of this is basically the same as Pukanszky's proof that the action of $\tilde{G}$ on $\hat{L}$ is countably separated. If the restriction of $\rho$ to $L$ is associated (via the Kirillov map) to the $L$-orbit of some $f \in I^{*}$, then the Auslander-Kostant machinery defines a $\tilde{G}$-equivariant Borel isomorphism 
from $\left\{h \in \mathfrak{I}^{*}:(h \mid \mathfrak{l} \in \tilde{G} f\} / K_{0}\right.$ onto $F_{0}$. $\left(K_{0}\right.$ and $\tilde{G}$ act on $\mathfrak{I}^{*}$ and $\mathfrak{I}^{*}$ via the coadjoint representation.) Hence $F_{0} / \tilde{G}$ is naturally Borel isomorphic to a Borel subset of $\mathfrak{f}^{*} / \tilde{G}$. But since $\operatorname{Ad}_{t}(\tilde{G})$ is connected and algebraic, $\mathfrak{f}^{*} / \tilde{G}$ is countably separated by a theorem of Chevalley-see [30, Remark 1.3] for references and more details. Hence the action of $\tilde{G}$ on $F_{0}$ is countably separated and $\tilde{G} \rho$ is closed. It follows that $\mathscr{Q}_{0} \subseteq \tilde{G} \rho$ and that some connected subgroup $H \supseteq G$ of $\tilde{G}$ acts transitively on $\mathscr{Q}_{0}$ (by the conjugation action of $\tilde{G}$ on the dual space of the normal subgroup $K_{0}$ ); the group $B_{0}$ defined earlier is then a quotient group of $H$.

Next we observe that for $a \in \tilde{G}$ and $x \in \mathfrak{f}$ (the Lie algebra of $K_{0}$ ), $\operatorname{ad}_{\mathfrak{q}} x$ and $\operatorname{ad}_{t}((\operatorname{Ad} a) x)$ have the same spectrum. This follows from the fact that if we realize $\mathfrak{g}$ and $\mathfrak{f}$ as linear Lie algebras in a suitable $g l(V), V$ a finitedimensional real vector space, then the adjoint action of $\tilde{G}$ on $f$ is defined by the conjugation action of a suitable subgroup of $\mathrm{GL}(V)$. Thus $\tilde{G}$ permutes the roots $[2, \mathrm{p} .1]$ of $\mathfrak{H}$, and since $\tilde{G}$ is connected, it actually leaves them invariant. (This will force $\tilde{G}$ to leave invariant a function $P$ which we will define shortly.) In particular, $\tilde{G}$ leaves invariant the function $j$ on $\mathfrak{f}$, where

$$
j(x)=\left|\operatorname{det}\left(\left(1-\exp \left(-\operatorname{ad}_{\mathfrak{t}} x\right)\right) / \operatorname{ad}_{\mathfrak{t}} x\right)\right|,
$$

and leaves invariant the open subset $\mathscr{V}=\left\{x \in \mathfrak{f}: \operatorname{ad}_{t} x\right.$ has no eigenvalue in $2 \pi i \mathrm{Z}-\{0\}\}$ of $\mathrm{f}$.

Let $f \in \mathscr{D}\left(K_{0}\right)^{+}$have its support in $\exp (\mathfrak{V})$, let $a \in \operatorname{Ad}(\tilde{G})$, and again let $\rho \in \mathbb{Q}_{0}$. Define $\alpha \in \mathscr{D}(\mathfrak{V})$ by $\alpha(x)=j(x) f(\exp x)$; then by [2, Remarque IX. 3.1.3, p. 249],

$$
(a \cdot \rho)(f)=(a \cdot \rho)(\alpha)=\int_{t} \alpha(x)(a \cdot \rho)(\exp x) d x .
$$

We evaluate the trace of this expression by means of Duflo's character formula [2, Theorème IX. 3.1.1]. First of all, we note that since $G$ is solvable, our original representation $\sigma$ may be associated with an element $g \in g^{*}$ (choose one in the appropriate orbit). Then $K_{0}$ is exactly $\left(G_{g}\right)_{0} L$ [29, Proposition I.6.1, p. 503]. The representation $\rho$ of $K_{0}$ may be associated with $h=g \mid \mathfrak{f} \in \mathfrak{f}^{*}$, and clearly $K_{0}=\left(K_{0}\right)_{h} L$, so by [2, Remarque IX. 3.1.5], the character formula is indeed applicable to $\rho$. Let $O$ be the orbit of $h$ in ${ }^{*}$, let $\beta$ be the canonical measure on $O$, and let $P=P_{O}$ as in the character formula be taken to be a product of functions $\sinh (\lambda / 2) /(\lambda / 2)$, where $\lambda$ ranges over certain roots of $\mathrm{f}$. We deduce that

$$
\operatorname{Tr}((a \cdot \rho)(f))=\operatorname{Tr}\left(\rho\left(a^{-1} \cdot f\right)\right)=\int_{O}\left(\left(a^{-1} \cdot \alpha\right) / P\right)^{\wedge}(y) d \beta(y),
$$

where ${ }^{\wedge}$ denotes the Fourier transform, $\left(a^{-1} \cdot \alpha\right)(x)=\alpha((\operatorname{Ad} a) x) \Delta(a)^{-1}$, and $\Delta$ is the factor by which $\tilde{G}$ multiplies Haar measure on $K_{0}$. Letting $\psi(a)=$ $\operatorname{Tr}((a \cdot \rho)(f))$, we can also use the fact that $\tilde{G}$ leaves $j$ and $P$ invariant to write 


$$
\psi(a)=\int_{O}(\alpha / P)^{\wedge}(a \cdot y) d \beta(y) .
$$

Recall that a certain connected subgroup $H \supseteq G$ of $\tilde{G}$ acts transitively on $\mathbb{Q}_{0}$, and that viewing $\psi$ as a function on $B_{0}=H / H_{\rho}$, we have $\psi \in L^{1}\left(B_{0}\right)$. We would like to deduce from this that $\psi$ (as a function on $B_{0}$ ) vanishes at infinity. First note that since $\tilde{G}$ is solvable and locally algebraic, it is the semidirect product of a vector group $V$ and a nilpotent group $U$, where $U \supseteq[\tilde{G}, \tilde{G}]=L$. The adjoint action of $V$ on $\tilde{\mathfrak{g}}$ (hence the coadjoint action on $\tilde{\mathfrak{g}}^{*}$ and $\left.\mathfrak{f}^{*}\right)$ is diagonalizable over the complex numbers, while the action of $U$ on ${ }^{*}$ is unipotent. (For the structure of solvable affine algebraic groups, see [5, Theorem 10.6].) Hence the action of any $a \in V$ on $\mathrm{f}^{*}$ is, with suitable choice of coordinates in $\mathrm{L}^{*}$, via multiplication of the $i$ th coordinate of a point

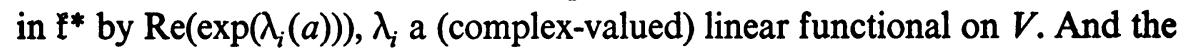
map $U \times \mathfrak{f}^{*} \rightarrow \mathfrak{t}^{*}$ given by the unipotent action of $U$ is a polynomial map.

We use all this information together with (1) for $\psi$ and the fact that $(\alpha / P)^{-}$ vanishes rapidly at infinity to show that $\psi$ is sufficiently "smooth" on $B_{0}$ so as to vanish at infinity. Let $s$ be a positive real number, and view $\psi$ as a function on $\tilde{G} / \tilde{G}_{\rho}$, a quotient group of $\tilde{G} / L \cong(U / L) \times V$. Since $K_{0}=\left(K_{0}\right)_{h} L, O$ is actually an orbit of the unipotent action of the nilpotent group $L$ on $\mathfrak{f}^{*}$; hence $O$ is closed in ${ }^{*}$ and there is a bijective polynomial map from some real vector space onto $O$ that carries Lebesgue measure to $\beta[2, \mathrm{I}$. 3.7, pp. 6-7]. The same arguments as in the proof of [31, Lemma 31] show that $\psi(a) \rightarrow 0$ for $a$ tending to infinity in $U / U_{\rho}$. Let $a=u v \in \tilde{G} / L$, with $u \in$ $(U / L), v \in V$, and the image of $a$ in a vector subgroup of $\tilde{G} / \tilde{G}_{\rho}$. Calculation using (1) shows that as $s \rightarrow \infty, \psi(s a)$ tends either to infinity exponentially (if enough of the $\operatorname{Re} \lambda_{i}(v)$ are large in absolute value and negative) or else to some finite limit. We see in this way that if $\tilde{G} / \tilde{G}_{\rho}$ is compactified into the product of a closed ball and a torus by adding limit points corresponding to the various rays through the origin in a maximal vector subgroup of $\tilde{G} / \tilde{G}$, then $\psi$ extends naturally to a function on this compactification, again called $\psi$, with values in $S^{2}=\mathrm{C} \cup\{\infty\}$. Consider the restriction of this function to the closure $B_{0}{ }^{\prime}$ of $B_{0}$. If $\psi$ assumes the value $\infty$ on the boundary of $B_{0}{ }^{\prime}$, then it actually takes this value on an open subset of the boundary (since if $\operatorname{Re} \lambda_{i}\left(v_{0}\right)>0$, then $\operatorname{Re} \lambda_{i}(v)>0$ for all $v$ near $v_{0}$ ), and this clearly contradicts the fact that $\psi \in L^{1}\left(B_{0}\right)$. So $\psi$ cannot grow exponentially on any ray in $B_{0}$, and (1) for $\psi$ then shows that $\psi$ must behave at infinity on $B_{0}$ no worse than a polynomial function. Since a polynomial function on a real vector space extends continuously to a projective space compactification, it follows that $\psi$ is continuous on $B_{0}{ }^{\prime}$. Then since a metric on $B_{0}$ giving rise to our compactification is dominated by the ordinary Euclidean metric, $\psi$ is uniformly continuous for the Euclidean metric on $B_{0}$. Since $\psi \in L^{1}\left(B_{0}\right)$ and 
$B_{0}$ is the direct product of a torus and a vector group, we conclude that $\psi$ must vanish at infinity on $B_{0}$.

Suppose $\mathbb{C}_{0}$ is not closed in $\hat{K}_{0}$. Then we can choose a sequence $\left\{a_{n}\right\}$ of elements of $B_{0}$ and some $\rho^{\prime} \in \hat{K}_{0}-\mathbb{C}_{0}$ with $a_{n} \cdot \rho \rightarrow \rho^{\prime}$. If the sequence $\left\{a_{n}\right\}$ were bounded in $B_{0}$, then we could choose a compact subset $C$ of $H$ containing representatives for all the $a_{n}$ 's. Since $\rho$ extends an irreducible representation of $L$, and since $L$ is nilpotent and thus CCR, $\rho$ is a CCR representation. (This may be seen directly but also follows from a more general result of Gootman [16, Corollary 3.2].) Thus $\{\rho\}$ is a closed point in $\hat{K}_{0}$, and $C \cdot \rho$ is closed in $\hat{K}_{0}$ by [23, Lemma 4.1]. Since $H \cdot \rho=\mathbb{Q}_{0}, \rho^{\prime} \in \mathbb{Q}_{0}$, a contradiction.

So, passing to a subsequence, we may suppose $a_{n} \rightarrow \infty$ in $B_{0}$. By the above, for $f \in \mathscr{D}\left(K_{0}\right)^{+}$with support in $\exp (\mathfrak{V}), \operatorname{Tr}\left(\left(a_{n} \cdot \rho\right)(f)\right) \rightarrow 0$. But the function $\rho \mapsto \operatorname{Tr}(\rho(f))$ is lower semicontinuous on $\hat{K}_{0}$ [8, Proposition 3.5.9], so for such $f$ s, $\operatorname{Tr}\left(\rho^{\prime}(f)\right)=0$. Since $\left\{f \in \mathscr{D}\left(K_{0}\right)^{+}: \operatorname{supp} f \subseteq \exp (\mathfrak{V})\right\}$ contains a bounded approximate identity for $C^{*}\left(K_{0}\right)$, lower semicontinuity of $x \mapsto$ $\operatorname{Tr}\left(\rho^{\prime}(x)\right)$ on $C^{*}\left(K_{0}\right)^{+}$implies that $\operatorname{Tr}\left(\rho^{\prime}(x)\right)=0$, and hence $\rho^{\prime}(x)=0$, for all $x \in C^{*}\left(K_{0}\right)^{+}$. This is clearly absurd, so $\mathbb{Q}_{0}$ is closed in $\hat{K}_{0}$.

We use this information as in the proofs of [31, Lemma 30] and [23, Theorem 2] to prove that $J$ is a maximal ideal of $C^{*}(G)$. Let $J_{1}$ be another primitive ideal of $C^{*}(G)$ with $J_{1} \supseteq J$-we will show that $J_{1}=J$. Choose an irreducible representation $\sigma_{1}$ of $G$ with $\operatorname{ker} \sigma_{1}=J_{1}$; then $\sigma_{1}$ is weakly contained in $\sigma$. The restriction of $\sigma_{1}$ to $K_{0}$ is supported on a $G$-orbit closure in $\operatorname{Prim}\left(K_{0}\right)$-let $Q$ be the inverse image of this set in $\hat{K}_{0}$. By continuity of restriction for the hull-kernel topologies [13, Lemma 5.1], $Q \subseteq \mathbb{Q}_{0}^{-}$, the ${ }^{-}$ denoting closure in $\hat{K}_{0}$. But we just saw that $\mathbb{Q}_{0}$ is closed in $\hat{K}_{0}$, so $Q \subseteq \mathbb{Q}_{0}$, proving, in particular, that $E\left(J_{1}\right)=E(J)$. As in the proof of [23, Theorem 2], our conclusion follows from the fact that $F / \Sigma$ is Hausdorff.

3.9. Corollary (CF. [8, Proposition 18.4.1]). Let $G$ be a connected unimodular solvable Lie group, $\sigma$ an S.I.S.S. factor representation of G. Then ker $\sigma$ is a closed point of $\operatorname{Prim}(G)$.

Proof. We may take $\sigma$ to be a subrepresentation of the left regular representation $\lambda$ of $G$, and since $\lambda$ is associated with the trace $\tau$, where $\tau(f)=f(e)$ for $f \in \mathcal{K}(G)$, it is clear that $\sigma(f)$ is generalized Hilbert-Schmidt even for all $f \in \mathcal{K}(G)$. Thus the theorem applies directly when $G$ is simply connected.

In the general case, let $H$ be the universal covering group of $G$ and let $p$ : $H \rightarrow G$ be the covering map. Also let $\tilde{\sigma}=\sigma \circ p$, which is an S.I.W.S. representation of $H$. Choose an open neighborhood $V$ of $e$ in $H$ small enough so that $p \mid V$ is a diffeomorphism. Then for $f \in \mathscr{D}(V), \tilde{\sigma}(f)=\sigma\left(f^{\circ} p^{-1}\right)$ is of generalized trace class. For a general $f \in \mathcal{D}(H)$, let $C$ be the support of $f$. We 
can choose finitely many elements $a_{1}, \ldots, a_{n} \in H$ such that $a_{1} V, \ldots, a_{n} V$ cover $C$, and by a routine partition of unity argument, $f$ is the sum of $n$ translates of functions in $\mathscr{D}(V)$. But if $\tilde{\sigma}(g)$ is of generalized trace class, then so is $\tilde{\sigma}(a \cdot g)=\tilde{\sigma}(a) \tilde{\sigma}(g)$ for any $a \in H$, since the trace class operators are an ideal in $\tilde{\sigma}(H)^{\prime \prime}$. Hence $\tilde{\sigma}(f)$ is a generalized trace class operator and the theorem shows that ker $\tilde{\sigma}$ is closed in $\operatorname{Prim}(H)$. However we have a natural homeomorphism $i$ of $\operatorname{Prim}(G)$ onto a closed subset of $\operatorname{Prim}(H)$ satisfying $i(\operatorname{ker} \sigma)=\operatorname{ker} \tilde{\sigma}$, so $\operatorname{ker} \sigma$ is a closed point in $\operatorname{Prim}(G)$.

3.10. Corollary. Let $G$ be a connected unimodular solvable Lie group, $\sigma$ an S.I.W.S. factor representation of G. Then $\mathrm{ker} \sigma$ is a closed point of $\operatorname{Prim}(G)$.

Proof. Let $Z$ be the center of $G$. As we saw earlier, $\sigma$ may be identified with an S.I.S.S. factor representation $\pi$ of some central extension $H$ of $G / Z$ by $T$. Now if $G$ is unimodular, so is $G / Z$, and hence so is $H$. Furthermore, $H$ is clearly solvable if $G$ is. So by Corollary $3.9, \operatorname{ker} \pi$ is a closed point of $\operatorname{Prim}(H)$. As in the last part of the proof of Corollary 3.9, this shows that ker $\sigma$ is a closed point of $\operatorname{Prim}(G)$.

3.11. We conclude this section by mentioning some examples (not claimed to be new) of nonnilpotent solvable Lie groups with square-integrable representations of various sorts, showing that the theorems of this section are not vacuous. The best known is the " $a x+b$ " group, the simply connected solvable group $G$ with Lie algebra $g=\operatorname{R} e_{1}+\operatorname{Re} e_{2}$, where $\left[e_{1}, e_{2}\right]=e_{2}$. This group has two S.I.S.S. irreducible representations associated with the two open $G$-orbits in $\mathrm{g}^{*}$; the condition of Theorem 3.5 obviously holds since $G$ has trivial center and acts freely on these orbits. For an example of a group with S.I.W.S. but not S.I.S.S. representations, one can consider the universal covering group of the "complex $a x+b$ " group; the Lie algebra of this group is the complexification of $\mathfrak{g}$ as just defined. In this case the dual of the Lie algebra contains an open dense orbit on which the adjoint group acts freely; however the group has center isomorphic to the integers.

For an example of a group with a non-type I S.I.S.S. factor representation (in fact a type $\mathrm{II}_{\infty}$ factor regular representation), let $\mathfrak{I}$ be a 4-dimensional real abelian Lie algebra with basis $e_{1}, \ldots, e_{4}$, let $\mathbf{R} e_{5}+\mathbf{R} e_{6}+\mathbf{R} e_{7}$ be a 3-dimensional abelian Lie algebra, and let $g$ be the semidirect product of these with nonvanishing brackets

$$
\begin{gathered}
{\left[e_{5}, e_{1}\right]=e_{2},\left[e_{5}, e_{2}\right]=-e_{1},\left[e_{5}, e_{3}\right]=\theta e_{4},} \\
{\left[e_{5}, e_{4}\right]=-\theta e_{3} \quad(\theta \text { irrational }), \quad\left[e_{6}, e_{1}\right]=e_{1},\left[e_{6}, e_{2}\right]=e_{2},} \\
{\left[e_{7}, e_{3}\right]=e_{3},\left[e_{7}, e_{4}\right]=e_{4} .}
\end{gathered}
$$

Let $G$ be the corresponding simply connected solvable group, which contains the Mautner 5-dimensional non-type I group as a normal subgroup. It is 
easily verified that $\mathrm{g}^{*}$ contains dense nonlocally closed $G$-orbits which satisfy the conditions of Theorem 3.4. ( $Z$ here is trivial.)

\section{The case of exponential solvable groups.}

4.1. Using Theorem 3.5, we can extend practically all of the remaining results of [24] to arbitrary exponential solvable Lie groups (which are always type I). Most of these results have been obtained by Duflo and Rais [11], but because our methods are rather different than theirs and because the conclusions illustrate and amplify the results of $\S 3$, it seems to us worthwhile to include them here. Let $G$ be a connected, simply connected, exponential solvable Lie group with center $Z$; then $Z$ is connected as is the stabilizer $G_{g}$ of any point in $\mathrm{g}^{*}$ (see for instance [2, Corollaire 3.4, p. 5]). Thus, as when $G$ is nilpotent, the S.I.W.S. irreducible representations of $G$ are obtained from precisely those $g \in g^{*}$ such that $G_{g}=Z$, or equivalently, $g_{g}=z$. As in [24], let us form, for $g \in g^{*}$, the Pfaffian $P(g)$ of the two-form $b_{g}$ on $g / z$ defined by the formula $b_{g}(\dot{x}, \dot{y})=g([x, y])(x, y \in g$; the dots indicate images modulo $z$ ). We have $g_{g}=z$ if and only if $b_{g}$ is nondegenerate or, equivalently, $P(g) \neq 0$, and this is an algebraic condition on $g$. In particular, if $G$ has one S.I.W.S. irreducible representation, then $P$ is a nonzero polynomial, and $\left\{g \in g^{*}: P(g) \neq 0\right\}$ is a nonempty Zariski open subset of $g^{*}$. Recall that for exponential groups, there is a natural bijection from $\mathrm{g}^{*} / G$ onto $\hat{G}[2$, Chapter VI] which is obviously a Borel map, hence a Borel isomorphism $\left(g^{*} / G\right.$ and $\hat{G}$ are standard Borel spaces). The following lemma is well known, but we include a proof for the sake of completeness.

4.2. LemMa. If $G$ is an exponential solvable group (as above), the measure class on $\hat{G}$ defined by the canonical decomposition of the left regular representation $\lambda$ corresponds to the image in $\mathrm{g}^{*} / G$ of the Lebesgue measure class on the real vector space $\mathrm{g}^{*}$.

Proof. We proceed by induction on the dimension of $G$. To begin the induction, we note that the result is trivial when $G$ is abelian. So assume $\operatorname{dim} G>1$ and the result is known for groups of dimension < $\operatorname{dim} G$. Let $H$ be a connected normal subgroup of $G$ of codimension 1 (such an $H$ always exists), and let $\lambda_{H}$ be the left regular representation of $H$. We know that $\lambda=\operatorname{Ind}_{H \uparrow G} \lambda_{H}$ and, by induction, $\lambda_{H} \approx \int_{\mathfrak{b}^{*}}^{\oplus} \pi_{f} d f$, where $\approx$ denotes quasiequivalence, $\pi_{f}$ denotes the representation of $H$ associated with $f \in \mathfrak{h}^{*}$ (which can be constructed so that $f \mapsto \pi_{f}$ is measurable), and $d f$ is Lebesgue measure on the vector space $\mathfrak{h}^{*}$. Hence $\lambda \approx \int_{\mathfrak{h}^{*}}^{\oplus}\left(\operatorname{Ind}_{H \uparrow G} \pi_{f}\right) d f$; we must show that $\lambda \approx \int_{\mathfrak{G}^{*}}^{\oplus} \pi_{g} d g$, with notation analogous to that for $H$.

Now for $f \in \mathfrak{h}^{*}$, the stability group in $G$ of $\pi_{f} \in \hat{H}$ is connected (since $G$ is exponential and $\mathfrak{h}^{*}$ is a $G$-module of exponential type [2,p.4]), hence equal to $H$ or $G$. Hence by the usual Mackey subgroup analysis, either $\operatorname{Ind}_{H \uparrow G} \pi_{f}$ is 
irreducible or else $\pi_{f}$ extends to an irreducible representation of $G$. (The Mackey obstruction vanishes since $G / H \cong R$.) In either case it is easy to see that $\operatorname{Ind}_{H \uparrow G} \pi_{f} \approx \int_{V}^{\oplus} \pi_{g+v} d v$, where $g \in \mathfrak{g}^{*}$ extends $f, V$ is the annihilator of $\mathfrak{h}$ in $g^{*}$, and $d v$ is Lebesgue measure on $V$. Combining this with the above, $\lambda \approx \int_{\mathfrak{h}^{*}}^{\oplus} \int_{V}^{\oplus} \pi_{s(f)+0} d f d v$, where $s: \mathfrak{h}^{*} \rightarrow \mathfrak{g}^{*}$ is a linear cross-section, and so $\lambda \approx \int_{g^{*}}^{\oplus} \pi_{g} d g$. (We have omitted checks of the measurability of various maps-these we leave to the reader. More details of this sort of argument may be found in [34].)

4.3. Proposition. Let $G$ be a connected, simply connected, exponential solvable Lie group. If $P$ is the zero polynomial, $G$ has no S.I.W.S. irreducible representations. Otherwise, the Plancherel measure class in $\hat{G}$ is concentrated on the set of S.I.W.S. irreducible representations.

Proof. This follows from the discussion above and a combination of the lemma with the fact that the zero set of a nonvanishing polynomial function on a vector space has measure zero.

4.4. The idea of the following proposition is part of the "folklore," and is attributed by C. C. Moore to J. Brezin and perhaps others. (For the case of $G$ nilpotent, this proposition has just appeared in the text of Brezin's lecture at the 1974 Marseille-Luminy Conference on Non-Commutative Harmonic Analysis, Lecture Notes in Math., no. 466, Springer, Berlin and New York, 1975.)

Proposition. Let $G$ be a connected, simply connected, exponential solvable Lie group. Let $O$ be an orbit of $G$ on $\mathrm{g}^{*}$, and let $\pi$ be the corresponding irreducible representation of $G$. Then the following are equivalent:

(i) $O$ is "flat," i.e., $O$ is an open subset of some affine subspace $A$ of $g^{*}$.

(ii) There exists a connected normal subgroup $H$ of $G$ such that $\pi$ is the composition with the canonical map $G \rightarrow G / H$ of an S.I.W.S. representation $\sigma$ of $G_{1}=G / H$.

Proor. (a) Suppose (ii) holds. Then by the discussion above, $\sigma$ is associated with an orbit $O_{1}$ of $G_{1}$ on $g_{1}^{*}$, such that $Z_{1}=Z\left(G_{1}\right)$ is the stabilizer of any point in $O_{1}$. Since $O_{1}$ must be contained in some translate $A_{1}$ of the annihilator of $z_{1}$ in $g_{1}^{*}$, while $O_{1}$ is locally closed and of the same dimension as $A_{1}, O_{1}$ is open in $A_{1}$. Now $O$ is clearly the image of $O_{1}$ under the adjoint of the canonical map $\mathrm{g} \rightarrow \mathrm{g}_{1}$, so (i) follows.

(b) Assume (i), and write $A=g+\mathfrak{l}^{\perp}$, where $\mathfrak{f}^{\perp}$ is the annihilator in $\mathfrak{g}^{*}$ of some subspace $\mathfrak{f}$ of $\mathfrak{g}$. Then $\mathfrak{t}$ is in fact an ideal of $\mathfrak{g}$, since $f$ must be the stability subalgebra of any point in $O$. Having chosen $g \in O$, let $\mathfrak{h}=\mathfrak{l} \cap$ $\operatorname{ker}(g)$. Then $[\mathfrak{g}, \mathfrak{h}] \subseteq[\mathfrak{g}, \mathfrak{f}] \subseteq \mathfrak{f} \cap \operatorname{ker}(g)=\mathfrak{h}$, since $\mathfrak{f}$ is an ideal and $g([\mathfrak{g}, \mathfrak{f}])$ $=g\left(\left[\mathfrak{g}, \mathfrak{g}_{\mathfrak{g}}\right]\right)=0$. So $\mathfrak{h}$ is an ideal of $\mathfrak{g}$-let $H$ be the corresponding normal subgroup of $G$. It is clear that $\pi$ is trivial on $H$, and thus comes from a 
representation $\sigma$ of $G_{1}=G / H$. It remains to show that $\sigma$ is S.I.W.S. For this we may replace $g$ by $g_{1}$ and assume $f \cap \operatorname{ker}(g)=(0)$. But then the calculation above shows that $[g, \mathfrak{f}]=(0)$; hence $f$ is central. Since $f$ is a stability subalgebra, it contains all of the center $z$ of $g$, proving that $f=z$ and thus that $P(g) \neq 0$. So $\sigma$ is S.I.W.S., and (ii) is proved.

4.5. Next we specialize to the case when $G$ is unimodular (and still exponential). Then by Corollary 3.10, the kernel of any S.I.W.S. irreducible representation $\pi$ of $G$ is a maximal ideal of $C^{*}(G)$, and the representation is CCR. (For all this we need only the easy argument that 3.8 implies 3.10, not the proof of 3.8 (which is unnecessary for type I groups).) Hence by Theorem 1 of [28], the orbit $G g$ in $g^{*}$ corresponding to $\pi$ is closed. Since $G g$ is contained in $g+z^{\perp}\left(z^{\perp}\right.$ the annihilator of $z$ in $\left.g^{*}\right)$ and has the same dimension, $G g=g+z^{\perp}$. Now the arguments of most of $\S \S 2$ and 3 of [24] apply without change; we merely summarize the results. First, however, we need a purely algebraic fact about Lie algebras, which was kindly communicated to the author by Professor M. Vergne.

4.6. Lemma. Let $k$ be a field of characteristic zero, and let $g$ be a finite dimensional Lie algebra over $k$. Let $U(\mathrm{~g})$ be the universal enveloping algebra of $\mathrm{g}$, let $K(\mathrm{~g})$ be its division ring of fractions, and let $C(\mathrm{~g})$ be the center of $K(\mathrm{~g})$. Let $R\left(g^{*}\right)^{\mathfrak{g}}$ denote the field of rational functions on $\mathrm{g}^{*}$ (the $k$-dual of $\mathrm{g}$ ) which are invariant under the coadjoint action of $\mathrm{g}$. Then there exists a canonical isomorphism of $C(\mathrm{~g})$ onto $R\left(\mathrm{~g}^{*}\right)^{\mathrm{g}}$.

Proof (M. Vergne). This was proved in [35] when $k$ is algebraically closed. Let $\bar{k}$ be an algebraic closure of $k$, let $\overline{\mathfrak{g}}=\mathrm{g} \otimes_{k} \bar{k}$, and let $\psi: C(\overline{\mathrm{g}}) \rightarrow R\left(\overline{\mathrm{g}}^{*}\right)^{\mathrm{g}}$ be the canonical isomorphism. We have to prove that $\psi$ sends $C(g)$ onto $R\left(g^{*}\right)^{g}$. So let $G$ be the Galois group of $\vec{k}$ over $k$. For $\sigma \in G$, and for $u=u_{1} u_{2}^{-1} \in C(\mathfrak{g})\left(u_{1}, u_{2} \in U(\mathfrak{g})\right)$ and $f \in \bar{g}^{*}$, we have by definition of $\psi$, $u_{1}-\psi(u)(f) u_{2} \in J(f)$ (notation as in [35]). Then $u_{1}^{\sigma}-(\psi(u)(f))^{\sigma} u_{2}^{\sigma} \in$ $J(f)^{\sigma}$, but $J(f)^{\sigma}=J\left(f^{\sigma}\right)$ by independence of polarizations, so that $(\psi(u)(f))^{\sigma}$ $=\psi\left(u^{\sigma}\right)\left(f^{\sigma}\right)$. Hence $\psi$ commutes with the action of $G$ and $\psi$ maps $C(\mathrm{~g})$ into $R\left(g^{*}\right)^{g}$.

On the other hand, suppose $r \in R\left(g^{*}\right)^{\text {g }}$. Then $r=\psi(u)$ for some $u \in C(\mathfrak{g})$. Since $r$ is $G$-invariant, so is $u$. But it is easy to see that this forces $u \in C(\mathbb{g}) \cap$ $K(\mathrm{~g})=C(\mathrm{~g})$.

4.7. THEOREM. Let $G$ be a connected, simply connected, unimodular, exponential solvable Lie group with center $Z$. Let $\mathrm{g}, P$, etc., be as above.

(a) Let $\pi$ be an irreducible representation of $G$ corresponding to the orbit $O=G g$ in $g^{*}$. Then the following are equivalent:

(i) $\pi$ is S.I.W.S. 
(ii) $P(g) \neq 0$, or equivalently, $b_{g}$ is nondegenerate on $\mathrm{g} / \mathfrak{z}$.

(iii) $O=g+z^{\perp}$.

(iv) $\operatorname{Ind}_{Z \uparrow G} \chi$ is primary, where $\chi$ is the element of $\hat{Z}$ of which $\pi \mid Z$ is a multiple.

Furthermore, $P$ depends only on the restriction $f$ of $g$ to $z$, and so may be viewed as a polynomial function on $z^{*}$ or as an element of $S(z)$, the symmetric algebra on $z$.

(b) There is a natural bijection $\phi$ from $\left\{f \in z^{*}: P(f) \neq 0\right\}$ onto the set of S.I.W.S. elements of $\hat{G}$, which is a homeomorphism for the usual topologies (relativized vector space topology and relativized Fell topology).

(c) Let $C$ (g) be as in 4.6. If $G$ has S.I.W.S. irreducible representations, then $C(\mathrm{~g})$ coincides with the field of fractions of $S(\mathrm{z})$. The converse holds if $G$ is further assumed to be locally algebraic.

Proof. Only (c) is not a direct copy of results from [24]. The proof of the first part of (c) is identical to the proof of the first half of Theorem 3 of [24], except that one must consider rational functions instead of polynomial functions. ( $C(\mathrm{~g})$ may be identified with the $G$-invariant rational functions on $g^{*}$ by 4.6.) The second half of (c) follows as in [24] from the fact that when $g$ is algebraic, the transcendence degree of $C(g)$ over $\mathbf{R}$ is equal to the codimension of a generic orbit of $G$ on $g^{*}$. (This fact was stated in [15], but no proof was given there. In any event, it follows from 4.6 as explained in [35] for the special case of an algebraically closed ground field.)

4.8. REMARKs. Note that if $G$ satisfies the hypotheses of 4.7 and if $G$ has S.I.W.S. representations, then $Z$ must be nontrivial, since $z=0$ would imply that $\hat{G}$ consists of a single point, by (iii) of (a) of the theorem. This is no longer true if one drops the hypothesis of unimodularity of $G$, as is shown by the familiar example of the " $a x+b$ " group. Secondly, we remark that (c) is formulated in terms of $C(\mathrm{~g})$ rather than in terms of the center of $U(\mathrm{~g})$, since the former may be strictly larger than the field of fractions of the latter when $\mathbf{g}$ is not nilpotent. (For instance, let $\mathrm{g}=\mathbf{R} e_{1}+\mathbf{R} e_{2}+\mathbf{R} e_{3}$, where $\left[e_{1}, e_{2}\right]=$ $e_{2},\left[e_{1}, e_{3}\right]=e_{3}$, and $\left[e_{2}, e_{3}\right]=0$. $U(\mathrm{~g})$ has trivial center, but $e_{2} e_{3}^{-1}$ is a nontrivial element of $C(\mathrm{~g})$.) It seems possible, although probably unlikely, that the assumption that $g$ be algebraic could be dropped in the second part of (c). (One certainly cannot allow $G$ to be nonunimodular and $g$ to be nonalgebraic and expect the result to be true-consider for instance the case $g=\mathbf{R} e_{1}+\mathbf{R} e_{2}+\mathbf{R} e_{3}$, where $\left[e_{1}, e_{2}\right]=e_{2},\left[e_{1}, e_{3}\right]=\lambda e_{3}$ with $\lambda$ irrational, $\left[e_{2}, e_{3}\right]=0$. Then $z$ and $C(g)$ are trivial, but $g^{*}$, being odd dimensional, cannot contain an open $G$-orbit.)

4.9. Proposition. Let $G, Z$, and $\phi$ be as in 4.7, and suppose $G$ has S.I.W.S. representations. Then the Plancherel measure of $G$, which by Proposition 4.3 is 
supported on the set of S.I.W.S. representations, is transported via $\phi$ to the measure $|P(f)|$ df on $z^{*}$, where df is Lebesgue measure on $z^{*}$, suitably normalized.

Proof. This is proved exactly the same way as Theorem 6 of [24], except that in place of the Kirillov-Pukanszky character formula for nilpotent groups, we must use the character formula and Plancherel theorem for exponential groups ([2, Chapter IX, §4] and [28]). (The "correction factor" depending on the roots of $g$ by which one must multiply before taking Fourier transforms is 1 in this case, and so may be omitted.)

4.10. Proposition. Let hypotheses and notation be as in Proposition 4.9. Then if $f \in z^{*}$ and $P(f) \neq 0$, the formal degree of $\phi(f)$ is just $|P(f)|$.

Proof. Let us remark first of all that this follows for almost all $f$ (with respect to Lebesgue measure on $z^{*}$ ) from Proposition 4.9 and the relation between formal degree and trace. But to avoid the possibility of an exceptional null set, we must be somewhat more careful. Perhaps one could prove this Proposition by a more elaborate version of the inductive proof of Theorem 4 of [24], but it seems easiest to modify the proof of Proposition 4.9. (In fact, Proposition 4.9 is actually a corollary of Proposition 4.10, but as its proof is slightly easier, we list it as a separate result. Similarly, Theorem 4 of [24] implies Theorem 6 of the same paper, except for the exact values of the normalizing constants.)

Fix $f \in z^{*}$ with $P(f) \neq 0$, and choose a lattice $\Gamma$ in $Z$ such that $f$ takes only integral values on $\log \Gamma$. Then $\phi(f)$ is trivial on $\Gamma$, and may be viewed as a representation $\pi$ of $G / \Gamma$. Since $G / \Gamma$ has compact center, $\pi$ is actually S.I.S.S., and by definition of the formal degree for S.I.W.S. representations, $\pi$ and $\phi(f)$ have the same formal degree $d_{\pi}$. (We are supposing that Haar measure $d s$ on $G / \Gamma$ is normalized so that if $d s$ is Haar measure on $G$ and $h \in \mathcal{K}(G)$, then

$$
\left.\int_{G} h(s) d s=\int_{G / \Gamma} \sum_{t \in \Gamma} h(s+t) d \dot{s} .\right)
$$

We compute $d_{\pi}$ by finding the discrete part of the Plancherel measure $\mu$ on $(G / \Gamma)^{\wedge}$. (Recall that by [8, Proposition 18.8.5], $\mu(\pi)=d_{\pi}$.)

First we note that virtually all of $[29, \mathrm{pp}$. 569-571] applies without essential change to our situation. In particular, there is a diffeomorphism $\omega: \mathrm{g} / \log \Gamma \rightarrow$ $G / \Gamma$ such that if $\psi: g \rightarrow g / \log \Gamma$ and $\Psi: G \rightarrow G / \Gamma$ are the respective canonical maps, then $\Psi \circ \exp =\omega \circ \psi$. Let $j$ be the absolute value of the Jacobian of $\omega ; j$ is $Z / \Gamma$-invariant and $j(0)=1$. Choose $\beta \in \mathscr{D}(G / \Gamma)$ with $\beta(e) \neq 0$ and define $\alpha \in \mathcal{D}(\mathrm{g} / \log \Gamma)$ by $\alpha(x)=j(x) \beta \circ \omega(x), x \in \mathrm{g} / \log \Gamma$. As in [2, Remarque IX. 3.1.3, p. 249], we have 


$$
\pi(\beta)=\int_{\mathbb{g} / \log \Gamma} \alpha(x) \pi \circ \omega(x) d x,
$$

and by substitution of the character formula for exponential groups ([2, Chapter IX] and [28]) for the character formula for nilpotent groups in the proof of [29, Lemma 2.2, pp. 570-571], we obtain the formula

$$
\operatorname{Tr} \pi(\beta)=\int_{O} \hat{\alpha}(l) d v_{g}(l) .
$$

Here $O$ is the $G$-orbit $g+z^{\perp}$ in $g^{*}$ ( $g$ any linear extension of $f$ ), viewed as a $G$-orbit in $(g / \log \Gamma)$, and $\nu_{g}$ is the "canonical measure" on $O$ obtained from $b_{g}$ in the usual way. Again as in the proofs of [24, Theorem 6] and Proposition 4.9 above, $d v_{g}(l)=|P(f)|^{-1} d l$ if $d l$ is Lebesgue measure.

Now suppose $\beta$ transforms according to the character

$$
z \mapsto \exp (2 \pi i f(\log z))
$$

under the action of $Z / \Gamma$ on $L^{2}(G / \Gamma)$. Then $\beta$ is in the $\pi$-primary subspace of $L^{2}(G / \Gamma)$, and so

$$
\begin{aligned}
\beta(e) & =\int_{(G / \Gamma)^{\varphi}} \operatorname{Tr}(\rho(\beta)) d \mu(\rho)=\mu(\pi) \operatorname{Tr}(\pi(\beta)) \\
& =d_{\pi} \operatorname{Tr} \pi(\beta)=d_{\pi}|P(f)|^{-1} \int_{O}^{\hat{\alpha}}(l) d l .
\end{aligned}
$$

However by the condition on $\beta, \hat{\alpha}$ is supported on $O$, so that the Plancherel theorem for the abelian Lie group $g / \log \Gamma$ gives

$$
\beta(e)=\alpha(0)=\int_{(\mathrm{g} / \log \Gamma)^{\hat{\alpha}}} \hat{\alpha}(l) d l=\int_{O}^{\hat{\alpha}(l) d l .}
$$

Comparing (2) and (3) for $\beta(e)$, we conclude that $d_{\pi}=|P(f)|$, proving the proposition.

4.11. Remark. The paper of Duflo and Raïs [11] contains a stronger result than 4.10, valid for nonunimodular groups, as well as the Plancherel formula for arbitrary exponential groups. The proof above was developed before we were aware of their work-we include it since the details are needed for the next theorem.

4.12. The last theorem of this section is an extension of Theorem 7 of [24]. Although the result may not be all that useful when $G$ is not nilpotent, it seems to us that the proof below is simpler than the one given by Moore and Wolf for the nilpotent case.

THEOREM. Let $G$ be a connected, simply connected exponential solvable Lie group with center $Z$, and let $H$ be a discrete subgroup of $G$ such that $G / H$ is compact. Assume that $G$ has S.I.W.S. irreducible representations. Then an S.I.W.S. irreducible representation $\pi$ of $G$ is contained in $\operatorname{Ind}_{H \uparrow G} 1$ (the regular representation of $G$ on $L^{2}(G / H)$ ) if and only if the restriction of $\pi$ to 
$\Gamma=H \cap Z$ is a multiple of the trivial representation. When $\pi$ is contained in Ind $_{H \uparrow G} 1$, its multiplicity is equal to its formal degree (as computed above in 4.10). (We assume Haar measures are normalized in a manner to be specified in the proof.)

Proof. Note first of all that $G$ is necessarily unimodular. Indeed, since $G / H$ carries a $G$-invariant measure, and since $H$ is unimodular, it follows that the modular function of $G$ is trivial when restricted to $H$. Hence $\operatorname{tr}(\operatorname{ad} x)=0$ for all $x \in \log H$. But it is clear that $\log H$ spans $g$, so $\operatorname{tr}(\operatorname{ad} x)$ $=0$ for all $x \in g$, and $G$ is unimodular. (See [19] for another proof of this.) In particular, 4.7 and 4.10 are applicable to $G$.

Observe next that $\Gamma$ is a lattice in $Z$, so that $G^{\prime}=G / \Gamma$ has compact center $Z^{\prime}=Z / \Gamma$. Let $H^{\prime}=H / \Gamma$. It will be convenient to work with $G^{\prime}$ in place of $G$. It is clear that $\operatorname{Ind}_{H \uparrow G} 1$ restricts to a multiple of the trivial representation on $\Gamma$, so that the given condition for containment of $\pi$ is necessary. So henceforth we assume $\pi$ is trivial on $\Gamma$, and view $\pi$ as a representation, which is actually S.I.S.S., of $G^{\prime}$. We must show then that $\pi$ is contained in $\operatorname{Ind}_{H^{\prime} \uparrow G^{\prime}} 1$ with multiplicity $N_{\pi}$ equal to its formal degree $d_{\pi}$. Recall that if $\mu$ is the Plancherel measure of $G^{\prime}, d_{\pi}$ is just $\mu(\{\pi\})$.

Suppose $\phi \in \mathscr{K}\left(G^{\prime}\right)$ is of positive type. Then by the trace formula for representations induced from discrete uniform subgroups [14, pp. 17-32], $\left(\operatorname{Ind}_{H^{\prime} \uparrow G^{\prime}} 1\right)(\phi)$ is of trace class and has trace given by

$$
\int_{G^{\prime} / H^{\prime}} \sum_{h \in H^{\prime}} \phi\left(g h g^{-1}\right) d \dot{g}=\sum_{\sigma \in \hat{G}^{\prime}} N_{\sigma} \operatorname{Tr} \sigma(\phi) .
$$

As in [2,pp. 250-252], this result holds for all $\phi \in \mathscr{D}\left(G^{\prime}\right)$, whether or not $\phi$ is of positive type. Let $\chi$ be the character of $Z^{\prime}$ determined by the restriction of $\pi$, and suppose $\phi \in \mathscr{D}\left(G^{\prime}\right)$ transforms according to $\chi$ under the action of $Z^{\prime}$ (by left translations). Then by the character formula for groups whose universal covering is exponential (as derived in the proof of Proposition 4.10), we see that $\operatorname{Tr} \sigma(\phi)$ vanishes for $\sigma \neq \pi$. Hence only the term $N_{\pi} \operatorname{Tr} \pi(\phi)$ contributes to the right-hand side of the formula above, and only the term involving $\pi$ contributes to the Plancherel inversion formula for $\phi$, so that

$$
\phi(e)=\int_{\hat{G}^{\prime}} \operatorname{Tr} \sigma(\phi) d \mu(\sigma)=d_{\pi} \operatorname{Tr} \pi(\phi) .
$$

Now $H^{\prime}$ is discrete in $G^{\prime}, Z^{\prime}$ is compact, and $H^{\prime} \cap Z^{\prime}=\{e\}$, so that we may choose a small $Z^{\prime}$-saturated neighborhood $V$ of $e$ in $G^{\prime}$ such that $V \cap H^{\prime}=\{e\}$. Also $G^{\prime} / H^{\prime}$ is compact, so that there exists a compact set $E$ in $G^{\prime}$ containing representatives for every point in $G^{\prime} / H^{\prime}$. Then we may choose a $Z^{\prime}$-saturated neighborhood $W$ of $e$ in $G^{\prime}$ such that $g^{-1} W g \cap H^{\prime}=$ $\{e\}$ for all $g \in E$. Suppose we can choose $\phi \in \mathscr{D}\left(G^{\prime}\right)$ transforming according 
to $\chi$ and supported in $W$. Then the trace formula above (assuming $G^{\prime} / H^{\prime}$ has measure 1) just reduces to

$$
N_{\pi} \operatorname{Tr} \pi(\phi)=\int_{G^{\prime} / H^{\prime}} \phi(e) d \dot{g}=\phi(e)=d_{\pi} \operatorname{Tr} \pi(\phi),
$$

so that if $\phi(e) \neq 0, N_{\pi}=d_{\pi}$ and our theorem is proved.

So we need only check that we can choose $\phi$ appropriately. But this is easy, for we can take $\phi$ of the form $\phi_{1} \phi_{2}$ (pointwise, not convolution, product), where $\phi_{1} \in \mathscr{D}(W), \phi_{2}$ is $C^{\infty}, \phi_{1}(e)$ and $\phi_{2}(e)$ are both nonzero, $\phi_{1}$ is constant on cosets of $Z^{\prime}$, and $\phi_{2}$ transforms according to $\chi$ under the action of $Z^{\prime}$. (To construct $\phi_{1}$, pull back a function in $\mathscr{D}(G / Z)$. For $\phi_{2}$, one may take a smooth coefficient function for $\pi$.)

4.13. Finally, to illustrate Theorem 4.7, we give an example of a unimodular exponential solvable group which has S.I.W.S. representations but which is not nilpotent. Let $\mathfrak{g}$ be the 5-dimensional Lie algebra over $\mathbf{R}$ with basis $e_{1}, \ldots, e_{5}$ and nonvanishing brackets $\left[e_{1}, e_{2}\right]=e_{3},\left[e_{5}, e_{1}\right]=e_{1},\left[e_{5}, e_{2}\right]=$ $-e_{2},\left[e_{5}, e_{4}\right]=e_{3}$.

This algebra is completely solvable; it is unimodular since $\operatorname{Tr}\left(\operatorname{ad} e_{5}\right)=1-$ $1=0$ and since $e_{1}, \ldots, e_{4}$ are ad-nilpotent. The center of $g$ is $z=\mathbf{R} e_{3}$. If $e_{1}^{*}, \ldots, e_{5}^{*}$ is a basis of $g^{*}$ dual to $e_{1}, \ldots, e_{5}$, it is easy to check that the stability subalgebra of $e_{3}^{*}$ is just $z$. Thus the simply connected analytic group $G$ with Lie algebra $g$ has square-integrable representations. The Pfaffian polynomial in this case is clearly $P\left(t e_{3}^{*}\right)=t^{2}$, so that $G$ has the same Plancherel measure (aside from perhaps a normalizing factor) as the 5dimensional Heisenberg group.

\section{The case of algebraic solvable groups over local fields.}

5.1. The characterization of the S.I.W.S. representations of type I solvable Lie groups given in Theorem 3.5 has analogues for $\mathfrak{p}$-adic solvable groups, which we outline briefly here. Let $k$ be a $p$-adic field (a nondiscrete totally disconnected locally compact field of characteristic zero) with ring of integers $\theta$, and assume for simplicity that the characteristic of the residue class field of $\mathcal{O}$ is not 2 . Let $\mathcal{G}$ be a connected solvable affine algebraic group defined over $k$ with unipotent radical $\mathcal{T}$, and let $\mathcal{T}$ be a maximal torus of $\mathcal{G}$ defined over $k$. Denote by $G, T$, and $N$ the groups of $k$-rational points of $\mathcal{G}, \mathcal{T}$, and $\Re$, respectively, so that $G$ is the semidirect product of the locally compact groups $T$ and $N$. Let $\mathfrak{g}, \mathrm{t}$, and $\mathfrak{n}$ denote the Lie algebras (over $k$ ) of $G, T$, and $N$ (resp.), and let $\mathfrak{g}^{*}, \mathrm{t}^{*}$, and $\mathfrak{n}^{*}$ be their $k$-duals. Howe [18] has studied the harmonic analysis of $G$ and of a certain open normal subgroup $C$ of $G\left(C_{\infty}\right.$ in his notation), where $C$ is homeomorphic (via the exponential map) to its "Lie algebra" $V . V$ is an $\theta$-sub-Lie algebra of $g$ containing $\mathfrak{n}$ and a compact open $\theta$-submodule of t. By [18, Proposition 1.1], $C$ is a liminary (CCR) group with 
$\hat{C}$ canonically homeomorphic to $\hat{V} / C$, and by [18, Theorem 1.1], $G$ is a type $I$ group whose dual (as a Borel space-Howe does not determine the topology of $\hat{G})$ is a fiber space over $\mathfrak{n}^{*} / G$, the fiber over an orbit $G f \in \mathfrak{n}^{*} / G$ looking like $\left(G_{f} N / N\right)^{*}$. (The action of $G$ on $\mathfrak{n}^{*}$ and of $C$ on $\hat{V}$ is the coadjoint representation.)

In the case where $G$ is unipotent (so that $C=G$ ), the S.I.W.S. representations of $G$ were observed by van Dijk [40] to be characterized by the same criteria as for nilpotent Lie groups. The natural generalization of this is the following.

5.2. Proposition. With notation as above, the S.I.W.S. representations of $C$ correspond to orbits $C \gamma \in \hat{V} / C$ such that the stabilizer $C_{\gamma}$ of $\gamma$ in $C$ is equal to the center $Z(C)$ of $C$.

Proof. If $z$ is the center of $g$, we clearly have $Z(C)=\exp (z \cap V)$. Suppose $\sigma \in \hat{C}$ corresponds to the orbit $C \gamma$, and let $\chi$ be the character of $Z(C)$ determined by the restriction of $\sigma$. Then $\gamma$ extends the character $\chi{ }^{\circ} \exp$ of $z \cap V$, and the orbit $C \gamma$ is contained in $\gamma+(z \cap V)^{\perp}$ (we use additive notation for $\hat{V}$ and denote annihilators in $\hat{V}$ by a ${ }^{\perp}$ ). Furthermore, minor modifications in the proof of Howe's Plancherel theorem for $C$ (see [18, Proposition 1.1] again) show that $\sigma$ is a subrepresentation of $\operatorname{Ind}_{z(C) \uparrow} \chi$ if and only if $C \gamma$ has positive measure in $\{\lambda \in \hat{V}: \lambda \mid(z \cap V)=\chi \circ \exp \}=\gamma+(z \cap$ $V)^{\perp}$ with respect to the translate of Haar measure on $(z \cap V)^{\perp}$. But $C \gamma$ is a closed submanifold of $(z \cap V)^{\perp}$ (viewing both as analytic manifolds over $k$ ), so it is easy to see that this happens if and only if $C_{\gamma}$ and $(z \cap V)^{\perp}$ have the same dimension (over $\theta$ ). Since $C$ is "exponential," this in turn happens if and only if $C_{\gamma}$ and $Z(C)$ have the same Lie algebra over $\theta$, and so are equal.

5.3. The corresponding analysis for $G$ is only slightly trickier. It does not seem possible (as in the Lie group case) to describe $\hat{G}$ in terms of $g^{*} / G$ in a natural way, primarily because the duals of the additive and multiplicative groups of $k$ look very different (unlike the situation over R). Nevertheless, we have the following analogue of Theorem 2 .

THEOREM. Let notation be as above. Fix $f \in \mathfrak{n}^{*}$, and let $g$ be any element of $g^{*}$ which extends $f$. Let $\sigma \in \hat{G}$, and suppose the restriction of $\sigma$ to $N$ is associated with the orbit $G f \in \mathfrak{n}^{*} / G$. Then $\sigma$ is S.I.W.S. if and only if $G_{g}$ (the stabilizer of $g$ for the coadjoint action of $G$ on $\mathrm{g}^{*}$ ) is compact modulo the center $Z$ of $G$.

Proof. All groups appearing in this proof will be algebraic (or more correctly, groups of $k$-rational points of algebraic groups defined over $k$ ), so that in the discussion below, connectedness and dimension are to be taken in the algebraic, not topological, sense. In particular, $H_{0}$ here denotes the 
connected component of the identity in the group $H$ for the Zariski $k$ topology.

To begin with, we note that if $\mathcal{G}$ contains a central subtorus of positive dimension, we may divide out by it, and hence we may suppose that $Z_{0} \subseteq N$. Let $\chi$ be the character of $Z_{0}$ determined by the restriction of $\sigma$. We know that $\sigma$ is S.I.W.S. if and only if $\sigma$ is a discrete summand of $\operatorname{Ind}_{z_{0} \uparrow} X$.

Let $\mu_{\chi}$ be a measure on $\hat{N}$ in the class determined by the canonical decomposition of $\operatorname{Ind}_{z_{0} \uparrow N} \chi$, and let $\pi$ be an element of $\hat{N}$ in the $G$-orbit $O$ where $\sigma \mid N$ lives. Since $\operatorname{Ind}_{Z_{0} \uparrow G} \cong \operatorname{Ind}_{N \uparrow G}\left(\operatorname{Ind}_{Z_{0 \uparrow N}} \chi\right)$, the same reasoning as for Lie groups shows that $\pi$ is S.I.W.S. if and only if the following two conditions hold:

(a) $O$ has positive $\mu_{\chi}$-measure, and

(b) $\left(G_{\pi} / N\right)^{\wedge}=\left(G_{f} N / N\right)^{\wedge}$ is discrete, or equivalently, $G_{f} N / N$ is compact. We shall show that these conditions are equivalent to the condition of the theorem.

First, note that as for real nilpotent groups, $\mu_{x}$ is equivalent to the image in $\hat{N}$ of (translated) Haar measure on the affine subspace $f+z^{\perp}$ in $\mathfrak{n}^{*}$, where $z^{\perp}$ is the annihilator in $\mathfrak{n}^{*}$ of the Lie algebra $z$ of $Z$. Furthermore, $G f$ is an algebraic subvariety of $f+z^{\perp}$ which is at least locally closed, so that (a) is equivalent to the condition that $G f$ have the same dimension as $f+z^{\perp}$, or that $\mathrm{g} / \mathfrak{g}_{f}$ and $\mathfrak{n} / \mathfrak{z}$ have the same dimension. Secondly, note that $G_{f}$ is algebraic, so that $\left(G_{f}\right)_{0}$ is the semidirect product of $N_{f}$ and a torus. Replacing $T$ by a conjugate torus if necessary, we may assume that this torus lies in $T$, so that $\left(G_{f}\right)_{0}=\left(N_{f} T_{f}\right)_{0}$. We claim that $\left(G_{g}\right)_{0}=\left(N_{g} T_{f}\right)_{0}$. For this it is enough to prove that for $x \in g_{f} \cap t, x \in g_{g}$. But for $x \in \mathfrak{l}$, ad $x$ has a complete set of eigenvectors in $\bar{g}=\mathfrak{g} \otimes_{k} \bar{k}$, for $\bar{k}$ an algebraic closure of $k$. Letting $\bar{f}, \bar{g}$ be the $\bar{k}$-linear extensions of $f, g$ (resp.) to $\overline{\mathfrak{n}}=\mathfrak{n} \otimes_{k} \bar{k}$ and $\bar{g}$, we see that if $x \in \mathrm{g}_{f} \cap \mathrm{t}, \bar{f}([x, y])=0$ for all $y \in \overline{\mathfrak{n}}$, and so $\bar{f}$ vanishes on all eigenvectors for ad $x$ in $\overline{\mathfrak{n}}$ with nonzero eigenvalue. But then $\bar{f}$ vanishes on all eigenvectors for ad $x$ in $\bar{g}$ with nonzero eigenvalue, since all such lie in $\overline{\mathfrak{n}}$, and so $x$ stabilizes $\bar{g}$ and hence lies in $g_{g}$.

Now assume (b), so that $T_{f}$ is compact (and thus anisotropic over $k$ ). By Lemma I.6.1 on p. 500 of [29], $\operatorname{dim}\left(G_{f} / G_{g}\right) \geqslant \operatorname{dim}\left(G / G_{f} N\right)$. (The lemma is stated for Lie groups, but the essence of the proof is straight Lie algebra theory, which is valid over any field of characteristic zero.) Assuming (a), $\operatorname{dim}\left(G / G_{f}\right)=\operatorname{dim}\left(N / Z_{0}\right)$, and combining this with the above,

$$
\begin{aligned}
\operatorname{dim}\left(G / G_{g}\right) & =\operatorname{dim}\left(G / G_{f}\right)+\operatorname{dim}\left(G_{f} / G_{g}\right) \\
& \geqslant \operatorname{dim}\left(N / Z_{0}\right)+\operatorname{dim}\left(G / G_{f} N\right)=\operatorname{dim}(G / Z)-\operatorname{dim} T_{f} .
\end{aligned}
$$

Since $Z_{0} \subseteq N \cap G_{g}$, and since $T_{f} \subseteq G_{g}$ by the remarks above, we see that $G_{g}$ 
contains $Z T_{f}$ and has the same dimension. Hence $G_{g} / Z T_{f}$ is finite, and $G_{g} / Z$ is compact.

Conversely, if $G_{g} / Z$ is compact, then clearly $\left(G_{g}\right)_{0}=\left(Z T_{f}\right)_{0}$ and $T_{f}$ is compact. This proves (b), and then $\operatorname{dim}\left(G_{f} / G_{g}\right)>\operatorname{dim}\left(G / G_{f} N\right)$ as before. The reverse inequality is trivial, so

$$
\begin{aligned}
\operatorname{dim}\left(G / G_{f}\right) & =\operatorname{dim}\left(G / G_{g}\right)-\operatorname{dim}\left(G_{f} / G_{g}\right) \\
& =\operatorname{dim}\left(T / T_{f}\right)+\operatorname{dim}\left(N / Z_{0}\right)-\operatorname{dim}\left(G_{f} / G_{g}\right)=\operatorname{dim}\left(N / Z_{0}\right),
\end{aligned}
$$

proving (a).

\section{REFERENCES}

1. L. Auslander and C. C. Moore, Unitary representations of solvable Lie groups, Mem. Amer. Math. Soc. No. 62 (1966), 199 pp. MR 34 \#7723.

2. P. Bernat et al., Représentations des groupes de Lie résolubles, Dunod, Paris, 1972.

3. B. Blackadar, Factor representations of restricted direct product groups, Ph.D. Dissertation, Univ. of California, Berkeley, 1975.

4. R. J. Blattner, On induced representations, Amer. J. Math. 83 (1961), 79-98. MR 23 \#A2757.

5. A. Borel, Linear algebraic groups, Benjamin, New York, 1969.

6. J. Brezin, Unitary representation theory for solvable Lie groups, Mem. Amer. Math. Soc. No. 79 (1968), 122 pp. MR 37 \#2896.

7. A. Connes and M. Takesaki, The flow of weights on factors of type III (preprint).

8. J. Dixmier, Les C*-algèbres et leurs représentations, 2nd ed., Gauthier-Villars, Paris, 1969. MR 39 \#7442.

9. Sur la représentation régulière d'un groupe localement compact connexe, Ann. Sci. École Norm. Sup. (4) 2 (1969), 423-436. MR 41 \#5553.

10. M. Duflo and C. C. Moore, On the regular representation of a nonunimodular locally compact group, J. Functional Analysis 21 (1976), 209-243.

11. M. Duflo and M. Rais, Sur l'analyse harmonique sur les groupes de Lie résolubles, Ann. Sci. École Norm. Sup. 9 (1976), 107-144.

12. E. G. Effros, $A$ decomposition theory for representations of $C^{*}$-algebras, Trans. Amer. Math. Soc. 107 (1963), 83-106. MR 26 \#4202.

13. J. M. G. Fell, Weak containment and induced representations of groups, Canad. J. Math. 14 (1962), 237-268. MR 27 \#242.

14. I. M. Gel'fand, M. I. Graev and I. I. Pyatetskii-Shapiro, Representation theory and automorphic functions, Saunders, Philadelphia, Pa., 1969. MR 38 \#2093.

15. I. M. Gel'fand and A. A. Kirillov, Sur les corps liés aux algèbres enveloppantes des algèbres de Lie, Inst. Hautes Études Sci. Publ. Math. No. 31, 1966, 5-19. MR 34 \#7731.

16. E. C. Gootman, Induced representations and finite volume homogeneous spaces, J. Functional Analysis 24 (1977), 223-240.

17. A. Guichardet, Caractères des algèbres de Banach inoolutives, Ann. Inst. Fourier (Grenoble) 13 (1962), 1-81. MR 26 \#5437; 30, p. 1203.

18. R. E. Howe, Topics in harmonic analysis on solvable algebraic groups (preprint).

19. R. R. Kallman, The existence of invariant measures on certain quotient spaces, Advances in Math. 11 (1973), 387-391. M.R 48 \#8682.

20. A. Kleppner, The structure of some induced representations, Duke Math. J. 29 (1962), 555-572. MR 25 \#5132.

21. R. Lipsman, Representation theory of almost connected groups, Pacific J. Math. 42 (1972), 453-467. MR 48 \#6317. 
22. C. C. Moore, Groups with finite dimensional irreducible representations, Trans. Amer. Math. Soc. 166 (1972), 401-410. MR 46 \#1960.

23. C. C. Moore and J. Rosenberg, Groups with $T_{1}$ primitive ideal spaces, J. Functional Analysis 22 (1976), 202-224.

24. C. C. Moore and J. Wolf, Square-integrable representations of nilpotent groups, Trans. Amer. Math. Soc. 185 (1973), 445-462. MR 49 \#3033.

25. J. Phillips, Positive integrable elements relative to a left Hilbert algebra, J. Functional Analysis 13 (1973), 390-409.

26. 83-92. , A note on square-integrable representations, J. Functional Analysis 20 (1975),

27. D. Poguntke, Der Raum der primitiven Ideale von endlichen Enweiterungen lokalkompakter Gruppen, Arch. Math. (Basel) 128 (1977), 133-138.

28. L. Pukanszky, On the unitary representations of exponential groups, J. Functional Analysis 2 (1968), 73-113. MR 37 \#4205.

29. , Unitary representations of solvable Lie groups, Ann. Sci. Ėcole Norm. Sup. 4 (1971), 457-608.

30. , Action of algebraic groups of automorphisms on the dual of a class of type I groups,

Ann. Sci. Ecole Norm. Sup. (4) 5 (1972), 379-396. MR 48 \# 466.

31. __ The primitive ideal space of solvable Lie groups, Invent. Math. 22 (1973), 74-118.

32. Characters of connected Lie groups, Acta Math. 133 (1974), 81-137. MR 50 \#13383.

33. _ Lie groups with completely continuous representations, Bull. Amer. Math. Soc. 81 (1975), 1061-1063.

34. S. R. Quint, Decomposition of induced representations of solvable exponential groups, $\mathrm{Ph} . \mathrm{D}$. dissertation, Univ. of California, Berkeley, 1975.

35. R. Rentschler and M. Vergne, Sur le semi-centre du corps enveloppant d'une algèbre de Lie, Ann. Sci. École Norm. Sup. 6 (1973), 389-405. MR 50 \# 13177.

36. M. A. Rieffel, Square-integrable representations of Hilbert algebras, J. Functional Analysis 3 (1969), 265-300. MR 39 \#6094.

37. __ Unitary representations of group extensions: an algebraic approach to the theory of Mackey and Blattner, Advances in Math. (to appear).

38. G. Schlichting, Eine Charakterisierung gewisser diskreter Gruppen durch ihre reguläre Darstellung, Manuscripta Math. 9 (1973), 389-409. MR 48 \#2302.

39. M. Smith, Regular representations of discrete groups, J. Functional Analysis 11 (1972), 401-406. MR 49 \#9641.

40. G. van Dijk, Square-integrable representations $\bmod Z$ of unipotent groups, Compositio Math. 29 (1974), 141-150. MR 51 \#814.

Department of Mathematics, University of California, Berkeley, California 94720

Current address: Department of Mathematics, University of Pennsylvania, Philadelphia, Pennsylvania 19174 